Issued by Sandia National Laboratories, operated for the United States Department of Energy by Sandia Corporation.

NOTICE: This report was prepared as an account of work sponsored by an agency of the United States Government. Neither the United States Government, nor any agency thereof, nor any of their employees, nor any of their contractors, subcontractors, or their employees, make any warranty, express or implied, or assume any legal liability or responsibility for the accuracy, completeness, or usefulness of any information, apparatus, product, or process disclosed, or represent that its use would not infringe privately owned rights. Reference herein to any specific commercial product, process, or service by trade name, trademark, manufacturer, or otherwise, does not necessarily constitute or imply its endorsement, recommendation, or favoring by the United States Government, any agency thereof, or any of their contractors or subcontractors. The views and opinions expressed herein do not necessarily state or reflect those of the United States Government, any agency thereof, or any of their contractors.

Printed in the United States of America. This report has been reproduced directly from the best available copy.

Available to DOE and DOE contractors from

Office of Scientific and Technical Information

P.O. Box 62

Oak Ridge, TN 37831

Prices available from (703) 605-6000

Web site: http://www.ntis.gov/ordering.htm

Available to the public from

National Technical Information Service

U.S. Department of Commerce

5285 Port Royal Rd

Springfield, VA 22161

NTIS price codes

Printed copy: A03

Microfiche copy: A01

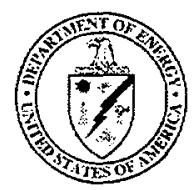




\section{DISCLAIMER}

Portions of this document may be illegible in electronic image products. Images are produced from the best available original document. 
SAND 99-2578

Unlimited Release

Printed October 1999

\title{
A Regularized Galerkin Boundary Element Method (RGBEM) for Simulating Potential Flow About Zero Thickness Bodies
}

\author{
Adrin Gharakhani, Sc.D. \\ Applied Scientific Research \\ adrin@Applied-Scientific.com \\ http://www.Applied-Scientific.com \\ 1800 East Garry Avenue, Suite 214 \\ Santa Ana, CA 92705
}

Sandia Contract AW-9960

\begin{abstract}
Potential flow about zero thickness bodies may be evaluated via the derivative boundary integral formulation of the Neumann problem describing the potential jump distribution on the body surface. For collocation-based methods, the boundary integral is shown to be singular unless the potential jump is at least $C^{1}$ continuous at the collocation points. In this report, a Regularized Galerkin Boundary Element Method (RGBEM) is formulated which relaxes the continuity constraint to $C^{0}$, and reduces the integrand singularity order by two. The new formulation identifies and removes the singular components of the integral equation mathematically, without resorting to complicated a posteriori singularity removal strategies that are common in the literature. The accuracy of RGBEM (with linear variation of the potential jump across planar triangular elements) is demonstrated to be superior compared to that of the constant-patch panel (or vortex loop) method, using the test problem of potential flow normal to a flat square plate.
\end{abstract}


Intentionally Left Blank 


\section{CONTENTS}

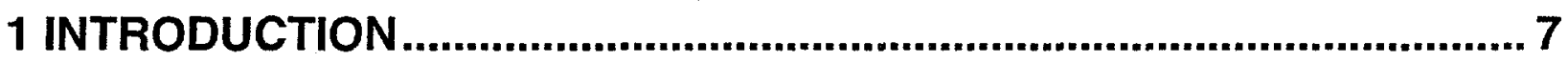

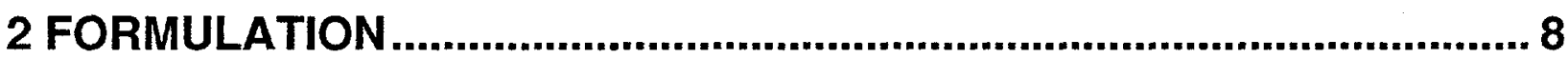

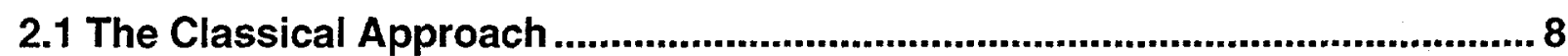

2.2 The Regularized Galerkin Boundary Element Method (RGBEM)....................11

2.3 RGBEM For Planar Triangular Elements ......................................................... 12

2.4 Evaluation Of $\int_{\partial \Omega_{k}} \int_{\partial \Omega_{m}} G d S_{m} d S_{k}$ For Planar Triangular Elements..................... 15

2.5 Evaluation Of The Velocity Field For Planar Triangular Elements ................. 18

3 RESULTS.......................................................................................... 19

SUMMARY ............................................................................................... 23

RECOMMENDATIONS........................................................................ 24

REFERENCES .................................................................................... 25

FIGURES ............................................................................................... 26

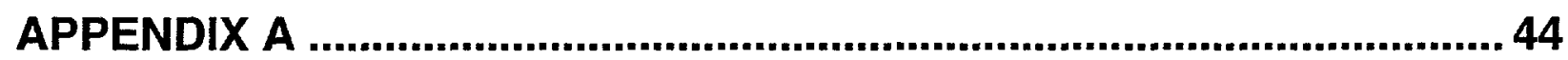

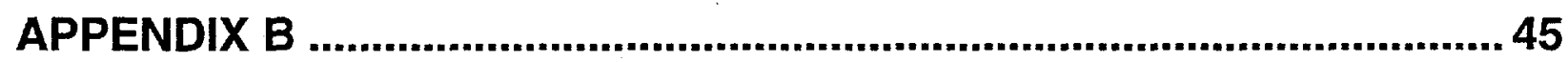

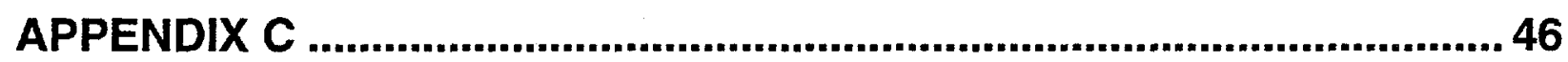


Intentionally Left Blank 


\section{INTRODUCTION}

The prediction of potential flow about zero thickness membranes by the boundary element method constitutes an integral component of the Lagrangian vortex-boundary element simulation of flow about parachutes. To this end, the vortex loop (or the panel) method has been used, for some time now, in the aerospace industry with relative success [1,2]. Vortex loops (with constant circulation) are equivalent to boundary elements with piecewise constant variation of the potential jump. In this case, extending the analysis in [3], the near field potential velocity evaluations can be shown to be $O(1)$ ! The accurate evaluation of the potential velocity field very near the parachute surface is particularly critical to the overall accuracy and stability of the vortex-boundary element simulations. As we will demonstrate in Section 3, the boundary integral singularities, which arise due to the application of low order boundary elements, may lead to severely spiked potential velocities at vortex element centers that are near the boundary. The spikes in turn cause the erratic motion of the vortex elements, and the eventual loss of smoothness of the vorticity field and possible numerical blow up.

In light of the arguments above, the application of boundary elements with (at least) a linear variation of the potential jump - or, equivalently, piecewise constant vortex sheets - would appear to be more appropriate for vortex-boundary element simulations. For this case, two strategies are possible for obtaining the potential flow field. The first option is to solve the integral equations for the (unknown) strengths of the surface vortex sheets. As we will discuss in Section 2.1, the challenge in this case is to devise a consistent system of equations that imposes the solenoidality of the locally 2-D vortex sheets. The second approach is to solve for the unknown potential jump distribution. In this case, for commonly used $C^{0}$ shape functions, the boundary integral is singular at the collocation points. Unfortunately, the development of elements with $C^{1}$ continuity for the potential jumps is quite complicated in 3-D. To this end, the application of Galerkin "smoothing" to the boundary integral equations removes the singularity at the collocation points; thus allowing the use of $C^{0}$ elements and potential jump distributions [4].

Successful implementations of the Galerkin Boundary Element Method to 2-D conduction [4] and elastostatic [5] problems have been reported in the literature. Thus far, the singularity removal algorithms have been based on a posteriori and mathematically complex reasoning, which have required Taylor series expansion and limit processes. The application of these strategies to 3-D is expected to be significantly more complicated.

In this report, we develop the formulation for a "Regularized" Galerkin Boundary Element Method (RGBEM). The regularization procedure involves simple manipulations using vector calculus to reduce the singularity of the hypersingular boundary integral equation by two orders for $C^{0}$ elements. For the case of linear potential jump distributions over plane triangles the regularized integral is simplified considerably to a double surface integral of the Green function! This is the case implemented and tested in this report. Using the example problem of flow normal to a square flat plate, the linear RGBEM predictions are demonstrated here to be more accurate, to converge faster, and to be significantly less spiked than the solutions obtained by the vortex loop method. 


\section{FORMULATION}

\subsection{The Classical Approach}

Let's assume that the free stream flow, with velocity $\bar{V}_{\infty}$, is perturbed due to the presence of a body $\Omega$, with boundary $\partial \Omega$. Then the boundary integral formulation prescribing the velocity potential $\Phi$ at any point $\vec{x}_{o}=\left(x_{o}, y_{o}, z_{o}\right)$ in the field is [6]

$$
\begin{aligned}
\Phi\left(\vec{x}_{o}\right)= & \int_{\partial \Omega} \vec{n}(\vec{x}) \cdot \vec{\nabla} G\left(\vec{x}, \vec{x}_{o}\right)\left(\Phi(\vec{x})-\Phi_{i}(\vec{x})\right) d S(\vec{x})- \\
& \int_{\partial \Omega} \vec{n}(\vec{x}) \cdot\left(\vec{\nabla} \Phi(\vec{x})-\vec{\nabla} \Phi_{i}(\vec{x})\right) G\left(\vec{x}, \vec{x}_{o}\right) d S(\vec{x})+\Phi_{\infty}\left(\vec{x}_{o}\right)
\end{aligned}
$$

where $G\left(\vec{x}, \vec{x}_{o}\right)=1 / 4 \pi\left|\vec{x}-\vec{x}_{o}\right|=1 / 4 \pi r$ is the Green function, $\vec{n}(\vec{x})=\left(n_{x}, n_{y}, n_{z}\right)$ is the unit normal to $\partial \Omega$ into the fluid, $d S(\vec{x})$ is the elemental surface area, $\Phi_{\infty}\left(\vec{x}_{o}\right)$ is the free stream velocity potential, and $i$ indicates the interior of the body.

Let's assume further a thin body and arbitrarily denote its upper and lower boundaries and their respective unit normals by $\left(\partial \Omega^{+}, n^{+}\right)$and $\left(\partial \Omega^{-}, n^{-}\right)$, respectively (see Fig. 1$)$. Then, Eq. (2.1) may be rewritten as

$$
\begin{aligned}
\Phi_{o} & =\int_{\partial \Omega^{+}} \vec{n}^{+} \cdot \vec{\nabla} G\left(\Phi^{+}-\Phi_{i}\right) d S-\int_{\partial \Omega^{+}} \vec{n}^{+} \cdot\left(\vec{\nabla} \Phi^{+}-\vec{\nabla} \Phi_{i}\right) G d S \\
& +\int_{\partial \Omega^{-}} \vec{n}^{-} \cdot \vec{\nabla} G\left(\Phi^{-}-\Phi_{i}\right) d S-\int_{\partial \Omega^{-}} \vec{n}^{-} \cdot\left(\vec{\nabla} \Phi^{-}-\vec{\nabla} \Phi_{i}\right) G d S+\Phi_{\infty}
\end{aligned}
$$

where the dependence of the parameters on $\vec{x}$ and $\vec{x}_{0}$ is not shown for the sake of simplicity of presentation.

In the limit of vanishing $\Omega$, where $\partial \Omega^{+} \rightarrow \partial \Omega^{-}$, Eq. (2.2) reduces to

$$
\Phi_{o}=\int_{\partial \Omega}[\Phi] \vec{n} \cdot \vec{\nabla} G d S-\int_{\partial \Omega} \vec{n} \cdot[\vec{\nabla} \Phi] G d S+\Phi_{\infty}
$$

where $\vec{n} \equiv \vec{n}^{+}=-\vec{n}^{-},[\Phi]=\Phi^{+}-\Phi^{-}$is the jump in the velocity potential across the thin body, and $\vec{n} \cdot[\vec{\nabla} \Phi]=\vec{n} \cdot \vec{\nabla} \Phi^{+}-\vec{n} \cdot \vec{\nabla} \Phi^{-}$is the corresponding jump in the normal flux, which is zero for impervious membranes or membranes allowing smooth fluid flux. Moreover, if we assume continuity of the velocity potential on $\partial \Omega$, then $\Phi^{+}=\Phi^{-}$at the intersection of $\partial \Omega^{+}$and $\partial \Omega^{-}$; or, equivalently, $[\Phi]=0$ on the closed contour around the open surface that defines the zerothickness membrane.

The velocity field at $\vec{x}_{0}$ due to the presence of an impervious open surface membrane, with boundary $\partial(\partial \Omega)$, in free stream is obtained by taking the gradient of Eq. (2.3): 


$$
\begin{aligned}
& \vec{u}_{o}=-\vec{\nabla}_{o} \Phi_{o}=-\bar{\nabla}_{o} \int_{\partial \Omega}[\Phi] \vec{n} \cdot \vec{\nabla} G d S+\vec{V}_{\infty} \\
& {\left.[\Phi]\right|_{\partial(\partial \Omega)}=0}
\end{aligned}
$$

where $\vec{u}_{o} \equiv \vec{u}\left(\bar{x}_{o}\right)$, and $\vec{\nabla}_{o}$ implies differentiation with respect to $\vec{x}_{o}$.

Equation (2.4a) is hypersingular due to the presence of $\vec{\nabla}_{0} \vec{\nabla} G$. However, the integral equation can be regularized by reformulating it into an equivalent integral equation that reduces the order of integrand singularity by one, as follows:

$$
\vec{u}_{o}=-\vec{\nabla}_{o} \times\left[\int_{\partial 2}(\vec{n} \times \vec{\nabla}[\Phi]) G d S-\oint_{\partial 2}[\Phi] G d \vec{s}\right]+\vec{V}_{\infty}
$$

where $d \vec{s}$ is the elemental vector tangent to the contour circumscribing the elemental surface area $d S$. See Appendix A for the derivation of (2.5).

Generally speaking, Eq. (2.5) does not (and cannot) reduce the singularity order of Eq. (2.4a); instead it shifts the singularity from the surface integral onto the contour integral. However, in the special case where $[\Phi]$ is continuous on the surface, the contour integral in (2.5) vanishes identically because of Eq. (2.4b). In this case, Eq. (2.5) provides a regularization (reduction in the singularity order) of Eq. (2.4a):

$$
\vec{u}_{o}=-\vec{\nabla}_{o} \times \int_{\partial 2}(\vec{n} \times \vec{\nabla}[\Phi]) G d S+\vec{V}_{\infty}
$$

Note that the new surface integral requires the evaluation of the tangential derivatives of the potential jump, $\vec{n} \times \vec{\nabla}[\Phi]$, which are readily available for a given distribution of [ $\Phi]$. Also note that Eq. (2.6) represents the velocity field due to a vortex sheet with strength $\vec{\gamma}=-\vec{n} \times \vec{\nabla}[\Phi]$.

Another special case of Eq. (2.5) pertains to the condition that $[\Phi]$ is piecewise constant on the surface, which implies that $\vec{n} \times \vec{\nabla}[\Phi] \equiv 0$ and that the surface integral vanishes identically. In this case, if the membrane surface is decomposed onto a set of $M$ contiguous patches, each associated with a constant distribution of the potential jump $[\Phi]^{m}$, Eq. (2.5) may be reduced to the following sum of integral contributions by the $M$ patches:

$$
\vec{u}_{o}=\sum_{m=1}^{M}\left\{[\Phi]^{m} \oint_{\partial \Omega_{m}} \vec{\nabla}_{o} G \times d \vec{s}_{m}\right\}+\vec{V}_{\infty}
$$

Note that $[\Phi]$ jumps in value on the edges of the patches and Eq. (2.7) is singular there. Also note that the term inside the braces represents the velocity due to a vortex loop with circulation $\Gamma_{m} \equiv[\Phi]^{m}$ around patch $m$ ! The vortex loop (or the panel) method has been used, for some time now, in the aerospace industry as a successful aerodynamics design tool $[1,2]$. However, its main drawback, as will be demonstrated in Section 3, is its low accuracy and slow 
rate of convergence. For this reason, we return to Eq. (2.6) and develop a higher order boundary element method for predicting potential flow about zero-thickness membranes.

A robust methodology for the solution of Eq. (2.6) in 3-D domains is not immediately apparent, even for the simplest case of a piecewise linear variation of $[\Phi]$ (or, equivalently, a piecewise constant variation of $\vec{\gamma}$ ). On the one hand, as we show in Appendix B, Eq. (2.6) with $[\Phi]$ as the unknown leads to a singularity at the collocation points, unless $[\Phi]$ is $C^{1}$ continuous there. Unfortunately, the development of elements with $C^{1}$ continuity for $[\Phi]$ is quite complicated in 3-D. On the other hand, the alternative of solving for $\vec{\gamma}$ as the unknown poses other challenges. This is because while $\vec{\gamma}$ is a locally 2-D vector tangent to the surface, its two components are constrained by the solenoidality condition $\vec{\nabla} \cdot \vec{\gamma}=0$. Therefore, only one component of $\bar{\gamma}$ is truly unknown in (2.6); the other component is, at least theoretically, available via the solenoidality constraint. Unfortunately, except for a few simplified cases, obtaining the second component directly via solenoidality is not straightforward [7]. To this end, two strategies have been proposed in the literature:

One strategy applies a " $-\vec{n}_{o} \times\left[\vec{n}_{o} \times(\cdot)\right] "$ operation to Eq. (2.6) and imposes tangential boundary conditions at the collocation points:

$$
\vec{n}_{o} \times \vec{\gamma}_{o}+\vec{n}_{o} \times\left[\vec{n}_{o} \times \int_{\partial \Omega} \vec{\gamma} \times \vec{\nabla}_{o} G d S\right]=\vec{n}_{o} \times\left[\vec{n}_{o} \times \vec{V}_{\infty}\right]
$$

where $\vec{\gamma}_{o} \equiv \vec{\gamma}\left(\vec{x}_{o}\right)$, and $\vec{n}_{o}$ is the unit normal to the surface at collocation point $\vec{x}_{o}$. This yields two sets of boundary integral equations for the two components of $\vec{\gamma}$ tangent to the surface [7]:

$$
\begin{array}{r}
\vec{\gamma}_{o} \cdot \vec{\rho}_{o}+\vec{\tau}_{o} \cdot \int_{\partial \Omega} \vec{\gamma} \times \vec{\nabla}_{o} G d S=\vec{\tau}_{o} \cdot \vec{V}_{\infty} \\
-\vec{\gamma}_{o} \cdot \vec{\tau}_{o}+\vec{\rho}_{o} \cdot \int_{\partial \Omega} \vec{\gamma} \times \vec{\nabla}_{o} G d S=\vec{\rho}_{o} \cdot \vec{V}_{\infty}
\end{array}
$$

where $\vec{\tau}_{o}=\left(\tau_{x}, \tau_{y}, \tau_{z}\right)_{o}$ and $\vec{\rho}_{o}=\left(\rho_{x}, \rho_{y}, \rho_{z}\right)_{o}$ are the unit tangents to the surface at $\vec{x}_{o}$. Note that for, at least, the case of a flat plate geometry, $\vec{\tau}_{o}$ and $\vec{\rho}_{o}$ may be arranged such that Eqs. (2.9a) and $(2.9 \mathrm{~b})$ become fully decoupled. This implies that $\vec{\gamma} \cdot \vec{\tau}$ and $\vec{\gamma} \cdot \vec{\rho}$ may be evaluated independently of each other, without enforcing the solenoidality constraint. Note that it is possible to complement Eqs. (2.9) with the solenoidality constraint. However, the resulting linear system of equations becomes over-specified and would have to be solved by least squares or similar error minimizing schemes. The lack of a good mechanism to impose solenoidality is the primary disadvantage of this approach.

An alternative strategy utilizes the solenoidality constraint and the normal component of Eq. (2.6) (to impose the normal flux boundary condition at the collocation points) as the two equations for obtaining the two components of $\vec{\gamma}$ : 


$$
\begin{aligned}
& \vec{n}_{o} \cdot \int_{\partial \Omega} \vec{\gamma} \times \vec{\nabla}_{o} G d S=\vec{n}_{o} \cdot \vec{V}_{\infty} \\
& \vec{\nabla} \cdot \vec{\gamma}=0
\end{aligned}
$$

where $\vec{n}_{o} \cdot \vec{u}_{o}=0$. Unfortunately, to the best of our knowledge, a consistent solution of this system of equations using classical boundary elements and shape functions for $\vec{\gamma}$ is not possible, although the method of least squares may be used with relative success to invert the system matrix.

\subsection{The Regularized Galerkin Boundary Element Method (RGBEM)}

In this report, we develop a Regularized Galerkin methodology for solving Eq. (2.6) with $[\Phi]$ as its unknown. The proposed formulation offers the following unique advantages over the previously mentioned solution strategies:

(1) Piecewise solenoidality of $\vec{\gamma}=-\vec{n} \times \vec{\nabla}[\Phi]$ is imposed implicitly for any polynomial variation of $[\Phi]$ within each boundary element,

(2) Only one equation, the normal component of (2.6), is necessary to obtain the one (and only) unknown $[\Phi]$,

(3) The Galerkin formulation relaxes the $C^{1}$ continuity requirement for $[\Phi]$ to $C^{0}$, while at the same time removing the integral singularity at the collocation points.

The starting point for the Galerkin formulation is the normal component of (2.6) and the application of the normal flux boundary condition:

$$
\vec{n}_{o} \cdot\left[\vec{\nabla}_{o} \times \int_{\partial \Omega}(\vec{n} \times \vec{\nabla}[\Phi]) G d S\right]=\vec{n}_{o} \cdot \vec{V}_{\infty}
$$

where $\vec{n}_{o} \cdot \vec{u}_{o}=0$ without loss of generality.

The Galerkin boundary element method attempts to minimize the discretization error in Eq. (2.11) in an integral sense as follows:

$$
\int_{\partial \Omega_{o}} \vec{n}_{o} \cdot\left[\vec{\nabla}_{o} \times \int_{\partial \Omega}(\vec{n} \times \vec{\nabla}[\Phi]) G d S\right] \psi_{o} d S_{o}=\int_{\partial \Omega_{o}} \vec{n}_{o} \cdot \vec{V}_{\infty} \psi_{o} d S_{o}
$$

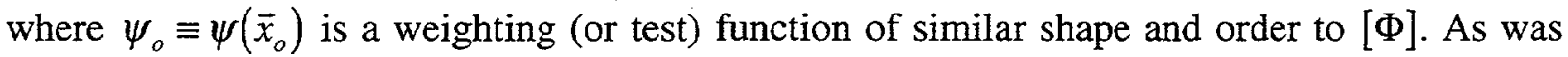

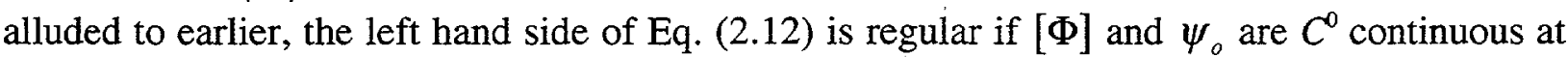
the collocation nodes. Note that although the singularities of (2.12) have been smoothed out by the application of the Galerkin method, in practice, special care is needed to remove them from the integration process. This has usually been accomplished in the literature by complex $a$ posteriori mathematical analysis and reasoning $[4,5]$. In the following, we propose a novel singularity removal strategy by manipulating Eq. (2.12) further and developing a "Regularized" 
Galerkin Boundary Element Method for solving the Neumann problem. The 2-D version of RGBEM can be derived directly from the 3-D formulation and is presented in Appendix C.

We begin by denoting the inner integral in (2.12) by a vectorial function $\vec{I}_{o}$, which depends only on $\vec{x}_{0}$. We focus on the left hand side of (2.12) and note the following identity:

$$
\int_{\partial \Omega_{o}} \psi_{o} \vec{n}_{o} \cdot \vec{\nabla}_{o} \times \vec{I}_{o} d S_{o}=\int_{\partial \Omega_{o}} \vec{n}_{o} \cdot \vec{\nabla}_{o} \times\left(\psi_{o} \vec{I}_{o}\right) d S_{o}-\int_{\partial \Omega_{o}} \vec{n}_{o} \cdot\left(\vec{\nabla}_{o} \psi_{o} \times \vec{I}_{o}\right) d S_{o}
$$

Application of the Stokes Theorem to the first integral on the right hand side of (2.13), and the vector identity $\vec{p} \cdot(\vec{q} \times \vec{r})=\vec{r} \cdot(\vec{p} \times \vec{q})$ to the second integral yields

$$
\int_{\partial \Omega_{o}} \psi_{o} \vec{n}_{o} \cdot \vec{\nabla}_{o} \times \vec{I}_{o} d S_{o}=\oint_{\partial \Omega_{o}} \psi_{o} \vec{I}_{o} \cdot d \vec{s}_{o}-\int_{\partial \Omega_{o}}\left(\vec{n}_{o} \times \vec{\nabla}_{o} \psi_{o}\right) \cdot \vec{I}_{o} d S_{o}
$$

Note that if $\psi_{o}$ is $C^{0}$ continuous at the collocation nodes then the contributions to the line integral above by neighboring elements cancel each other out identically. This derivation demonstrates explicitly how the application of the Galerkin method to (2.11) helps remove the source of singularity at the collocation points. Finally, substituting (2.14) into (2.12) yields the proposed Regularized Galerkin Boundary Element formulation for flow about membranes:

$$
\int_{\partial \Omega_{0}}\left(\vec{n}_{o} \times \vec{\nabla}_{o} \psi_{o}\right) \cdot\left[\int_{\partial \Omega}(\vec{n} \times \vec{\nabla}[\Phi]) G d S\right] d S_{o}=-\int_{\partial \Omega_{o}} \vec{n}_{o} \cdot \vec{V}_{\infty} \psi_{o} d S_{o}
$$

Equation (2.15) has reduced the singularity order of Eq. (2.4a) by two, from $\vec{\nabla}_{o} \vec{\nabla} G$ to $G$ ! In the process, the differentiation has been shifted from $G$ onto $[\Phi]$ and $\psi_{0}$ in the form of tangential gradients. This shift in the gradients further simplifies the process of evaluating the integrals. For example, for linearly varying $[\Phi]$ and $\psi_{o}$ over plane triangular elements, the tangential gradients are constants and (2.15) simplifies tremendously to

$$
\left(\vec{n}_{o} \times \vec{\nabla}_{o} \psi_{o}\right) \cdot(\vec{n} \times \vec{\nabla}[\Phi]) \int_{\partial \Omega_{o}} \int_{\partial \Omega} G d S d S_{o}=-\int_{\partial \Omega_{o}} \vec{n}_{o} \cdot \vec{V}_{\infty} \psi_{o} d S_{o}
$$

\subsection{RGBEM For Planar Triangular Elements}

The boundary element implementation of Eq. (2.16) begins by decomposing the surface of interest onto $M$ triangular elements, and assigning a local orthogonal coordinate system $(\xi, \zeta, \eta)^{m}$ and a corresponding unit basis vector $(\bar{\tau}, \vec{\rho}, \vec{n})_{m}$ to each element $m$, such that $(\xi, \zeta, \eta)^{m}=\left(\vec{x}_{m}-\vec{x}_{o}\right) \cdot(\vec{\tau}, \vec{\rho}, \vec{n})_{m} . \vec{x}_{m}$ denotes the global coordinates of any point on element $m$. The local coordinates are oriented such that $(\vec{\tau}, \vec{\rho})_{m}$ is parallel to the element plane, and $\vec{\tau}_{m}$ is along the longest (and first) side of the triangle - in the direction that is consistent with $\vec{n}_{m}$ and the right hand rule $[8,9]$. See Fig. 2 for a schematic diagram.

The linear variation of $[\Phi]^{m}$ and $\psi^{m}$ on element $m$ is prescribed by 
$f\left(\xi^{m}, \zeta^{m}\right)=a_{f}^{m}+b_{f}^{m} \xi^{m}+c_{f}^{m} \zeta^{m}$, where $f$ is a dummy variable denoting $[\Phi]$ or $\psi$. Furthermore,

$$
\begin{aligned}
& a_{f}^{m}=\frac{1}{2 S_{m}} \sum_{i=1}^{3}\left(\xi_{i+1}^{m} \zeta_{i-1}^{m}-\xi_{i-1}^{m} \zeta_{i+1}^{m}\right) f_{i}^{m} \\
& b_{f}^{m}=\frac{1}{2 S_{m}} \sum_{i=1}^{3}\left(\zeta_{i+1}^{m}-\zeta_{i-1}^{m}\right) f_{i}^{m} \\
& c_{f}^{m}=-\frac{1}{2 S_{m}} \sum_{i=1}^{3}\left(\xi_{i+1}^{m}-\zeta_{i-1}^{m}\right) f_{i}^{m}
\end{aligned}
$$

where $\left(\xi_{i}^{m}, \zeta_{i}^{m}\right)$ and $f_{i}^{m}=f\left(\xi_{i}^{m}, \zeta_{i}^{m}\right)$ are the coordinates and the nodal value of $f$ at node number $i$ of element $m$, respectively. $S_{m}$ is the surface area of element $m$. Note that index $i$ is circular, so that $i+1=1$ when $i=3$, and $i-1=3$ when $i=1$. The tangential gradients of $f$ are thus given by

$$
(\vec{n} \times \vec{\nabla} f)_{m}=-\left(f_{, \zeta}\right)^{m} \vec{\tau}_{m}+\left(f_{, \xi}\right)^{m} \vec{\rho}_{m}=-c_{f}^{m} \vec{\tau}_{m}+b_{f}^{m} \vec{\rho}_{m}
$$

Substituting Eq. (2.18) in the discretized form of Eq. (2.16) yields

$$
\sum_{k=1}^{M} \sum_{m=k}^{M}\left(-c_{\psi}^{k} \vec{\tau}_{k}+b_{\psi}^{k} \vec{\rho}_{k}\right) \cdot\left(-c_{[\Phi]}^{m} \vec{\tau}_{m}+b_{[\Phi]}^{m} \vec{\rho}_{m}\right) I_{m k}=-\sum_{k=1}^{M} \int_{\partial \Omega_{k}} \vec{n}_{k} \cdot \vec{V}_{\infty}^{k}\left(a_{\psi}^{k}+b_{\psi}^{k} \xi^{k}+c_{\psi}^{k} \zeta^{k}\right) d S_{k}
$$

where $I_{m k}=\int_{\partial \Omega_{k}} \int_{\partial \Omega_{m}} G d S_{m} d S_{k}$.

Assuming a piecewise-uniform free stream velocity $\vec{V}_{\infty}^{k}$ across each element $k$, and substituting Eqs. (2.17) into (2.19) yields

$$
\sum_{k=1}^{M} \sum_{m=k}^{M} G_{m k}\left(\begin{array}{l}
\vec{\tau}_{k} \cdot \vec{\tau}_{m} \sum_{j=1}^{3}\left(\xi_{j+1}^{k}-\xi_{j-1}^{k}\right) \psi_{j}^{k} \sum_{i=1}^{3}\left(\xi_{i+1}^{m}-\xi_{i-1}^{m}\right)[\Phi]_{i}^{m}+ \\
\vec{\rho}_{k} \cdot \vec{\rho}_{m} \sum_{j=1}^{3}\left(\zeta_{j+1}^{k}-\zeta_{j-1}^{k}\right) \psi_{j}^{k} \sum_{i=1}^{3}\left(\zeta_{i+1}^{m}-\zeta_{i-1}^{m}\right)[\Phi]_{i}^{m}+ \\
\vec{\tau}_{k} \cdot \vec{\rho}_{m} \sum_{j=1}^{3}\left(\xi_{j+1}^{k}-\xi_{j-1}^{k}\right) \psi_{j}^{k} \sum_{i=1}^{3}\left(\zeta_{i+1}^{m}-\zeta_{i-1}^{m}\right)[\Phi]_{i}^{m}+ \\
\vec{\rho}_{k} \cdot \bar{\tau}_{m} \sum_{j=1}^{3}\left(\zeta_{j+1}^{k}-\zeta_{j-1}^{k}\right) \psi_{j}^{k} \sum_{i=1}^{3}\left(\xi_{i+1}^{m}-\xi_{i-1}^{m}\right)[\Phi]_{i}^{m}
\end{array}\right)=-\frac{1}{3} \sum_{k=1}^{M} \sum_{j=1}^{3} \vec{n}_{k} \cdot \vec{V}_{\infty}^{k} S_{k} \psi_{j}^{k}
$$

where $G_{m k}=\frac{I_{m k}}{4 S_{m} S_{k}}$. 
Equation (2.20) is rewritten in the following compact format to depict the process of setting up the linear system of $N$ equations for the unknown [ $\Phi]$ at the $N$ collocation points on the surface:

$$
\begin{aligned}
& \sum_{k=1}^{M} \sum_{m=k}^{M} \sum_{i=1}^{3} \sum_{j=1}^{3} A_{i j}^{m k}[\Phi]_{i}^{m} \psi_{j}^{k}=\sum_{k=1}^{M} \sum_{j=1}^{3} B_{k} \psi_{j}^{k} \\
& A_{i j}^{m k}=\frac{\int_{\partial \Omega_{k}} \int_{\partial \Omega_{m}} G d S_{m} d S_{k}}{4 S_{m} S_{k}}
\end{aligned}\left(\begin{array}{l}
\vec{\tau}_{k} \cdot \vec{\tau}_{m}\left(\xi_{j+1}^{k}-\xi_{j-1}^{k}\right)\left(\xi_{i+1}^{m}-\xi_{i-1}^{m}\right)+ \\
\vec{\rho}_{k} \cdot \vec{\rho}_{m}\left(\zeta_{j+1}^{k}-\zeta_{j-1}^{k}\right)\left(\zeta_{i+1}^{m}-\zeta_{i-1}^{m}\right)+ \\
\vec{\tau}_{k} \cdot \vec{\rho}_{m}\left(\xi_{j+1}^{k}-\xi_{j-1}^{k}\right)\left(\zeta_{i+1}^{m}-\zeta_{i-1}^{m}\right)+ \\
\vec{\rho}_{k} \cdot \vec{\tau}_{m}\left(\zeta_{j+1}^{k}-\zeta_{j-1}^{k}\right)\left(\xi_{i+1}^{m}-\xi_{i-1}^{m}\right)
\end{array}\right)
$$

Now, if $\alpha=\alpha(i, m)$ is the global node number that the $i$-th node of element $m$ points to, and $\beta=\beta(j, k)$ is the number corresponding to the $j$-th node of element $k$, then $A_{\alpha \beta}=A_{\beta \alpha}$ represents an element of the assembled matrix $A$ at row (or column) $\alpha$ and column (or row) $\beta$. Similarly, $B_{\beta}$ represents element $\beta$ of the right hand vector of the linear system. With this notation, the linear system of equations looks like

$$
\left\{A_{\alpha \beta}[\Phi]_{\alpha}=B_{\beta}\right\} \psi_{\beta} \quad(\alpha, \beta)=1, \cdots, N
$$

Note that, unlike $[\Phi]_{\alpha}$ which represents the unknown potential jump at a collocation point $\alpha$, the nodal values of the Galerkin weight $\psi_{\beta}$ do not explicitly enter the final form of the linear system, since they appear on both sides of Eq. (2.22) as scaling factors and thus cancel out.

Three comments are in order here before a method is proposed for evaluating $I_{m k}$ :

First, $[\Phi]=0$ is known at collocation points on the boundary of the membrane surface. There are two options for incorporating this boundary information into the linear system of equations. In the first approach, (rows of) equations and matrix columns that correspond to nodes on the boundary are discarded and the reduced linear system is solved to obtain $[\Phi]$ at the interior nodes. In the second approach, the diagonal elements in the matrix that correspond to the boundary nodes are assigned a very large value, so that $[\Phi]=0$ is recovered as part of the solution. We use the latter for its simplicity of implementation.

Second, note that the summation over the $m$ elements in $(2.19-2.21)$ starts from element $k$. This is because - as verified by Eq. (2.16) - the system matrix is symmetric and only the upper 
(or lower) triangle of the matrix need be assembled explicitly. Thus, the symmetry of the Regularized Galerkin formulation cuts the cost of matrix assembly down by a factor of two.

Third, because of this matrix symmetry, the Conjugate Gradient Method can be used to invert the matrix instead of the Bi-Conjugate Gradient Method that is typically utilized in boundary element methods. This leads to a factor of two savings in CPU time.

\subsection{Evaluation Of $\int_{2 \Omega_{k}} \int_{\partial \Omega_{m}} G d S_{m} d S_{k}$ For Planar Triangular Elements}

In this section, we present a highly accurate methodology for evaluating the $I_{m k}$ integrals. In the process, we take advantage of the geometric simplicity of planar triangular elements and develop analytical solutions to the extent feasible. For (other) semi-analytical integrations we use a high order adaptive quadrature scheme with arbitrary precision control.

To begin, we note that a closed-form solution exists for the inner integral, the detailed derivation of which is given in [9]. The idea is to convert the area integral of the harmonic function into a line integral around the perimeter of the element using the divergence theorem. Since the integrals are carried out along each side $i$ of element $m$ around its perimeter, a local two-dimensional coordinate system $(p, q)_{m, i}$ is used in the $(\xi, \zeta)^{m}$ plane such that $p_{m, i}$ is parallel to side $i$ of element $m$ and $q_{m, i}$ is normal to it - as depicted in Fig. 3. The origin $\vec{x}_{o}$ of the coordinate system for element $m$ is an evaluation point on element $k$, and is thus the independent variable for the outer integral. The contour integral for element $m$ is given as follows $[8,9]$

$$
\begin{aligned}
& 4 \pi I_{m}=\int_{\partial \Omega_{m}} \frac{d S_{m}}{r_{m}}=-\sum_{i=1}^{3} \int_{p_{m, i}}^{2} p_{m, i} \frac{q_{m, i} r_{m, i} d p_{m, i}}{\left(r_{m, i}^{2}-\eta_{m}^{2}\right)}+\mid \eta_{m} \theta_{m} \\
& r_{m, i}^{2}=p_{m, i}^{2}+q_{m, i}^{2}+\eta_{m}^{2}
\end{aligned}
$$

where $\left({ }^{1} p_{m, i}, q_{m, i}\right)$ and $\left({ }^{2} p_{m, i}, q_{m, i}\right)$ are the coordinates of the beginning and end points of line segment $i$, respectively, and $\theta_{m}$ is a two-dimensional angle which accounts for the integral singularity when the evaluation point $\vec{x}_{o}$ falls into or on triangle $m . \theta_{m}=2 \pi$ if $\vec{x}_{o}$ falls inside triangle $m ; \theta_{m}=\pi$ if $\vec{x}_{o}$ falls on its perimeter, excluding the vertices; $\theta_{m}=0$ if $\vec{x}_{o}$ falls outside triangle $m$; and $\theta_{m}$ is equal to the angle of the vertex if $\vec{x}_{o}$ falls on the vertex. Note that in the present context we assign a subscript to $\eta$, rather than the usual superscript, to improve readability. The final form of (2.23) is as follows [9]:

$$
4 \pi I_{m}=\sum_{i=1}^{3}\left[\begin{array}{l}
\left|\eta_{m}\right| \operatorname{atan} 2\left(q_{m, i} \sqrt{p_{m, i}^{2}+q_{m, i}^{2}+\eta_{m}^{2}},\left|\eta_{m}\right| p_{m, i}\right)- \\
q_{m, i} \log \left(p_{m, i}+\sqrt{p_{m, i}^{2}+q_{m, i}^{2}+\eta_{m}^{2}}\right)
\end{array}\right]^{2 p_{m, i}}+\left|\eta_{m}\right|_{m} \quad \eta_{m} \neq 0
$$




$$
\begin{aligned}
& 4 \pi I_{m}=\sum_{i=1}^{3}\left[-q_{m, i} \log \left(p_{m, i}+\sqrt{p_{m, i}^{2}+q_{m, i}^{2}}\right)\right]_{p_{m, i}}^{2} p_{m, i} \quad \eta_{m}=0 \\
& \theta_{m}=\sum_{i=1}^{3}\left[\tan ^{-1} \frac{p_{m, i}}{q_{m, i}}\right]_{i p_{m, i}}^{2 p_{m, i}}
\end{aligned}
$$

We have not been able to derive a generalized closed-form formulation for the outer integral $I_{m k}=\int_{\partial \Omega_{k}} I_{m} d S_{k}$. For this purpose, we had to partially rely on available numerical integration algorithms. Since we were interested in maintaining controlled accuracy, we searched for triangle-based adaptive quadrature techniques with optimum error estimation capability. To this end, Netlib Algorithm 706 [10] was trimmed to fit the specific needs of the problem at hand and implemented in the code. The detailed description of Algorithm 706 is given in $[10,11,12]$ and is beyond the scope of this report. Very briefly, the algorithm applies a degree 13 symmetric quadrature rule that utilizes 37 optimally positioned evaluation points inside the triangle [12]. A globally adaptive strategy is applied whereby triangles with greatest estimated errors are repeatedly subdivided into four new subtriangles, and integrals and errors over the new subtriangles are estimated until either the user-specified error requirement is satisfied or the maximum allowable number of subtriangles have been created $[10,11]$.

The semi-analytic evaluation of $I_{m k}$, at some level of resolution that uses $v$ subtriangles, may be represented by the following:

$$
I_{m k}=\sum_{n=1}^{v} \sum_{i=1}^{37} w_{i} I_{m}\left(\vec{x}_{o}^{n, i}\right)
$$

where $w_{i}$ is the quadrature weighting at the $i$-th evaluation point, and $\vec{x}_{o}^{n, i}$ represents the global coordinates of the $i$-th evaluation point of the $n$-th subtriangle on element $k$. Note that $\vec{x}_{0}^{n, i}$ is used as the origin $\vec{x}_{o}$ when evaluating $I_{m}$.

The semi-analytical algorithm explained above works very well when the two triangles $m$ and $k$ are not contiguous. Generally, a maximum error of $10^{-10}$ is achievable before adaptation is invoked. However, for coincident $(m=k)$ and edge-sharing elements an inefficiently large number of subtriangles $v$ are necessary to evaluate the integrals at some nominal level of accuracy. This is because Eqs. (2.24) are logarithmic, which the standard quadrature weighting functions are ill-equipped to handle in cases where the evaluation points are too close to the elements. Fortunately, for the case of coincident elements we have derived the closed-form formulation for integral $I_{m k}$. Additionally, for the case of edge-sharing elements we can integrate out the contribution of the shared edge to $I_{m k}$ analytically, thus rendering the application of the quadrature algorithm to the remainder of the integral acceptable.

For the case of coincident elements, we consider the contributions by the element sides to Eq. (2.24b) separately, and integrate them over the element in the local coordinate system of the 
sides. The contribution by each side $i$ to $I_{m k}$ is denoted ${ }^{i} I_{m k}$ and is given below:

$$
\begin{aligned}
& 4 \pi I_{m k}=\frac{2 S_{k}^{2}}{3}\left\{\begin{array}{l}
\frac{{ }^{31} p_{k, i}{ }^{21} l_{k, i}}{{ }^{31} l_{k, i}^{3}} \log \left(\frac{{ }^{31} l_{k, i}{ }^{3}{ }^{31} p_{k, i}{ }^{21} l_{k, i}+{ }^{31} l_{k, i}{ }^{32} l_{k, i}}{{ }^{21} l_{k, i}\left({ }^{31} l_{k, i}-{ }^{31} p_{k, i}\right)}\right)-\frac{1}{{ }^{21} l_{k, i}} \log \left(\frac{{ }^{32} p_{k, i}+{ }^{32} l_{k, i}}{{ }^{31} p_{k, i}+{ }^{31} l_{k, i}}\right)- \\
\frac{{ }^{32} p_{k, i}{ }^{21} l_{k, i}}{{ }^{32} l_{k, i}^{3}} \log \left(\frac{{ }^{32} l_{k, i}^{2}{ }^{32} p_{k, i}{ }^{21} l_{k, i}{ }^{31} l_{k, i}{ }^{32} l_{k, i}}{{ }^{21} l_{k, i}\left({ }^{32} l_{k, i}+{ }^{32} p_{k, i}\right)}\right)+\frac{{ }^{32} l_{k, i}-{ }^{21} l_{k, i}}{{ }^{31} l_{k, i}^{2}}+\frac{{ }^{31} l_{k, i}-{ }^{21} l_{k, i}}{{ }^{32} l_{k, i}^{2}}
\end{array}\right\} \\
& { }^{21} p_{k, i}={ }^{2} p_{k, i}-{ }^{1} p_{k, i} \quad{ }^{31} p_{k, i}={ }^{3} p_{k, i}-{ }^{1} p_{k, i} \quad{ }^{32} p_{k, i}={ }^{3} p_{k, i}-{ }^{2} p_{k, i} \\
& { }^{21} l_{k, i}={ }^{21} p_{k, i} \quad{ }^{31} l_{k, i}=\sqrt{{ }^{31} p_{k, i}^{2}+\left({ }^{3} q_{k, i}-{ }^{1} q_{k, i}\right)^{2}} \quad{ }^{32} l_{k, i}=\sqrt{{ }^{32} p_{k, i}^{2}+\left({ }^{3} q_{k, i}-{ }^{2} q_{k, i}\right)^{2}}
\end{aligned}
$$

where $\left({ }^{1} p_{k, i},{ }_{k, i}\right)$ and $\left({ }^{2} p_{k, i},{ }^{2} q_{k, i}\right)$ are the (local) coordinates of the beginning and end points of segment $i$ of element $k$, respectively, and $\left({ }^{3} p_{k, i},{ }^{3} q_{k, i}\right)$ denotes the coordinates of the third node of the triangle. Note that the last two terms inside the braces cancel out when the contributions from all three sides are considered. The final form of $I_{k k} \equiv I_{m k}$ for coincident elements is

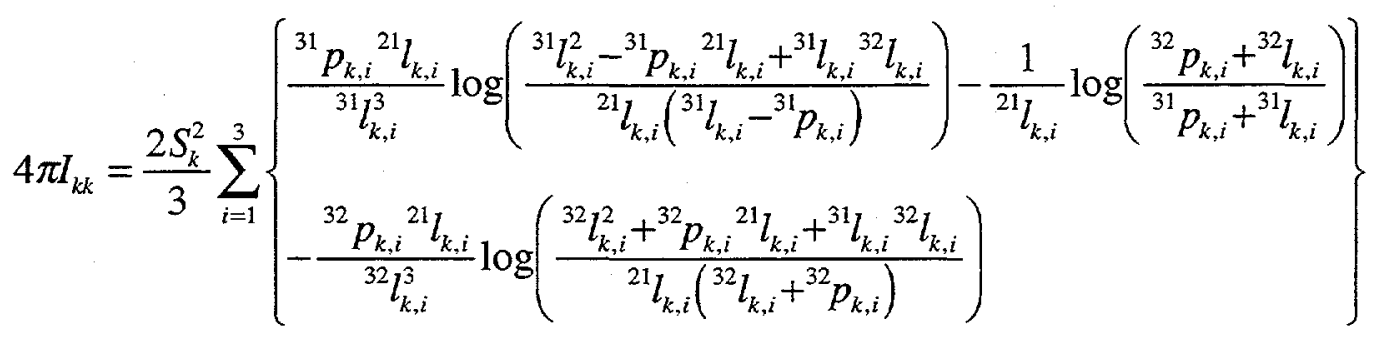

For the case of edge sharing elements, the logarithmic contribution by the shared edge to $I_{m k}$ may be evaluated by a slight modification of Eqs. (2.26), whereas the contributions by the remaining sides of element $m$ can be obtained semi-analytically:

$$
4 \pi I_{m k}=-\vec{n}_{k} \cdot \vec{n}_{m} \frac{2 S_{k}^{2}}{3}\left\{\begin{array}{l}
\frac{{ }^{31} p_{k, 1}{ }^{21} l_{k, 1}}{{ }^{31} l_{k, 1}^{3}} \log \left(\frac{{ }^{31} l_{k, 1}^{2}-{ }^{31} p_{k, 1}{ }^{21} l_{k, 1}+{ }^{31} l_{k, 1}{ }^{32} l_{k, 1} l_{k, 1}\left({ }^{31} l_{k, 1}{ }^{31} p_{k, 1}\right)}{{ }^{32} l_{k, 1}^{3}} \log \left(\frac{{ }^{32} l_{k, 1}^{2}+{ }^{32} p_{k, 1}{ }^{21} l_{k, 1}+{ }^{31} l_{k, 1}{ }^{32} l_{k, 1}}{{ }^{21} l_{k, 1}\left({ }^{32} l_{k, 1}+{ }^{32} p_{k, 1}\right)}\right)\right. \\
-\frac{1}{{ }^{21} l_{k, 1}} \log \left(\frac{p_{k, 1}+{ }^{32} l_{k, 1}}{{ }^{31} p_{k, 1}+{ }^{31} l_{k, 1}}\right)+\frac{{ }^{32} l_{k, 1}-{ }^{21} l_{k, 1}}{{ }^{31} l_{k, 1}^{2}}+\frac{{ }^{31} l_{k, 1}-{ }^{21} l_{k, 1}}{{ }^{32} l_{k, 1}^{2}}
\end{array}\right\}
$$




$$
\begin{aligned}
4 \pi^{2-3} I_{m}= & \sum_{i=1}^{3}\left[\left|\eta_{m}\right| \operatorname{atan} 2\left(q_{m, i} \sqrt{p_{m, i}^{2}+q_{m, i}^{2}+\eta_{m}^{2}},\left|\eta_{m}\right| p_{m, i}\right)\right]_{p_{m, i}}^{2 p_{m, i}} \\
& -\sum_{i=2}^{3}\left[q_{m, i} \log \left(p_{m, i}+\sqrt{p_{m, i}^{2}+q_{m, i}^{2}+\eta_{m}^{2}}\right)\right]_{p_{m, i}}^{2}+\mid \eta_{m} \theta_{m} \\
I_{m k}=I_{m k} & +\sum_{n=1}^{v} \sum_{i=1}^{37} w_{i}{ }^{2-3} I_{m}\left(\vec{x}_{o}^{n, i}\right)
\end{aligned}
$$

where side 1 is assumed to be the shared edge, and the dot product of the normals accounts for the fact that the two elements may not be coplanar.

\subsection{Evaluation Of The Velocity Field For Planar Triangular Elements}

Once the potential jump distribution on the $M$ boundary elements is known, Eq. (2.6) may be used to obtain the potential velocity in the fluid domain:

$$
\vec{u}_{o}=-\sum_{m=1}^{M} \int_{\partial \Omega_{m}}(\vec{n} \times \vec{\nabla}[\Phi])_{m} \times \vec{\nabla} G d S_{m}+\vec{V}_{\infty}
$$

For plane triangular elements, the integrals in (2.29) may be evaluated analytically [8,9]. Furthermore, for the linear variation of $[\Phi]^{m}$ considerable simplifications are possible:

$$
-\int_{\partial \Omega_{m}}(\vec{n} \times \vec{\nabla}[\Phi])_{m} \times \vec{\nabla} G d S_{m}=b_{[\Phi]}^{m} \eta^{m} I^{3} \vec{\tau}_{m}+c_{[\Phi]}^{m} \eta^{m} I^{3} \vec{\rho}_{m}-\left(b_{[\Phi]}^{m} I_{\zeta}^{3}+c_{[\Phi]}^{m} I_{\zeta}^{3}\right) \vec{n}_{m}
$$

where $I^{3}=\int_{\partial \Omega_{m}} \frac{1}{4 \pi r_{m}^{3}} d S_{m}, \quad I_{\xi}^{3}=\int_{\partial \Omega_{m}} \frac{\xi^{m}}{4 \pi r_{m}^{3}} d S_{m}$ and $I_{\zeta}^{3}=\int_{\partial \Omega_{m}} \frac{\zeta^{m}}{4 \pi r_{m}^{3}} d S_{m} \quad$ [8, 9]. Thus, the $i$-th component of the velocity at $\vec{x}_{o}$ is given by

$$
u_{i}\left(\vec{x}_{o}\right)=\sum_{m=1}^{M}\left[\eta^{m}\left(b_{[\Phi]}^{m} \tau_{m, i}+c_{[\Phi]}^{m} \rho_{m, i}\right) I^{3}-\left(b_{[\Phi]}^{m} I_{\xi}^{3}+c_{[\Phi]}^{m} I_{\zeta}^{3}\right) n_{m, i}\right]+V_{\infty, i}\left(\vec{x}_{o}\right) \quad i=1,2,3
$$




\section{RESULTS}

The robustness of RGBEM is examined parametrically using the test problem of potential flow normal to a square flat plate. The predictions are compared with results obtained by the vortex loop method (VLM) to demonstrate the superior accuracy of RGBEM as compared to VLM. In addition to the flat plate problem, preliminary results from the RGBEM simulation of potential flow about a hemispherical shell and a slotted hemispherical shell are presented.

The coordinates of the four vertices of the flat plate are assigned at $(0,0,0),(1,0,0),(1,1,0)$ and $(0,1,0)$. The free stream velocity is set to $(0,0,1)$. The plate surface is meshed uniformly in the $x$ and $y$ directions, and each square mesh is subdivided into two right triangles. Note that in the subsequent figures, the references to grid resolution correspond to square elements.

Figures $4 \mathrm{a}$ and $4 \mathrm{~b}$ depict the (absolute value of the) surface potential jump distribution along $(x, 0.5,0)$, obtained by RGBEM and VLM, respectively. We note that the lower resolution RGBEM predictions are within acceptable range of the high resolution result, and convergence is quite quick. In contrast, the VLM predictions display very poor accuracy at low resolutions, and as shown in Fig. 4a, the highest resolution VLM prediction is far from converged. Indeed, increasing the VLM resolution from $40 \times 40$ to $80 \times 80$ (not shown) reduces the peak potential jump from 0.709 to only 0.704 . We were not able to obtain results at higher resolutions due to limitations on core memory requirements. (We will leave the rigorous convergence studies to future publications.)

Figure 5 displays the potential velocity vector field in the vicinity of the plate, in its plane of symmetry. Figures $6 a$ and $6 \mathrm{~b}$ depict the potential velocities evaluated by RGBEM in the parallel and normal directions to the flat plate, respectively, along $(0.5 \leq x, y \leq 1, z=0.001) .501$ equidistant points were used in these and all subsequent figures. Additionally, the diagonal distance was normalized by $\sqrt{2}$. In Figs. $6, \bar{n} \times \vec{\nabla}[\Phi]$ was evaluated by directly differentiating the potential jump on the surface, and is thus piecewise constant across each triangular element. The consequence of this formulation, as verified in (6a), is that the tangential velocity in the fluid domain and proximal to the plate surface assumes a piecewise constant profile. Similarly, the normal velocity displays spikes where the corresponding tangential velocity experiences a jump. The spikes in the normal velocity manifest the existence of singularity at the collocation points, which the RGBEM formulation removes for matrix assembly purposes (only). Nevertheless, given the $C^{0}$ continuity condition for $[\Phi]$, the velocity profiles across most of the plate converge quite acceptably except within a narrow region (of one element size) around the boundary of the plate, where the accuracy of the predictions and the convergence rates are substandard. This may be because $[\Phi]$ varies as the square root of the distance to the edges and traditional polynomial shape functions may be inadequate for resolving the velocity profiles at the boundary (edges) [13]. Apparently, in order to achieve fast convergence, it may be necessary to implement special (quarter point) boundary elements in the boundary region [13]. It should be noted, however, that the tangential as well as the normal velocity components are infinite at the boundary edges on the surface, which negatively affect the accuracy of the near field velocity evaluations by any numerical method. This edge singularity is a mathematical reality that cannot be avoided without 
the introduction of some form of a physical model. Indeed, the formulation presented in this report breaks down at the edges because of the geometric singularity there, and the fact that the corresponding surface normal is undefined (or multiply defined).

The quality of the velocity profiles near the plate may appear far from ideal, until one evaluates the velocities using the traditional vortex loop method, as shown in Figs. $7 \mathrm{a}$ and $7 \mathrm{~b}$. In this case, we note that both the tangential and the normal components of the velocity display spikes. This is because $[\Phi]$ is piecewise constant and its gradients at the boundary element edges will naturally develop spikes. Indeed, since the normal flux boundary condition is satisfied at the element centroids, consistency requirements limit the choice of evaluation points in the fluid domain to points along the normal to the plate and through the element centroids only. This is clearly evident in Fig. $7 \mathrm{~b}$ where the normal velocity profile along an element side varies from a spike of one sign on one side (of the element) to a spike of the opposite sign on the other side (of the element), with the value at the center evaluated most accurately.

Figures $8 \mathrm{a}$ and $8 \mathrm{~b}$ compare the velocity profiles obtained by RGBEM and VLM along $(0.5 \leq x, y \leq 1, z=0.001)$. Note that even the low resolution (11x11) RGBEM profiles are substantially more accurate than the high resolution (40x40) VLM results. At $80 \times 80$ (not shown), VLM predictions are still far from resolved; e.g., the peak normal velocity is 3.76 for the $80 \times 80$ case compared to 6.17 for the $40 \times 40$ case. Note that the $11 \times 11$ (RGBEM) and the $80 \times 80$ (VLM) grid resolutions correspond to 200 and 12,482 triangular elements, respectively. This is a 62 -fold increase in the number of elements!

Figures $9 \mathrm{a}$ and $9 \mathrm{~b}$ represent the potential velocities evaluated by RGBEM in the parallel and normal directions to the flat plate, respectively, along $(0.5 \leq x, y \leq 1, z=0.01)$. The trend for the velocities at this higher elevation $(z=0.01)$ are similar to those nearer the plate at $z=0.001$ (Figs. 6). However, the step jumps and the spikes are smoother (and more benign) due to the fast decay of the influence of the sources on the plate. Note in Fig. $9 \mathrm{~b}$ that the overshoot in the normal velocity near the edge of the plate disappears at higher resolutions. This is further indication that the plate boundaries need special attention; i.e., either the grid distribution near the boundaries must be refined or non-standard boundary elements must be utilized there.

Figures $10 \mathrm{a}$ and $10 \mathrm{~b}$ display a comparison of the velocity profiles obtained by RGBEM and VLM along $(0.5 \leq x, y \leq 1, z=0.01)$. Again, while they are considerably smoother at $z=0.01$, the high resolution ( $40 \times 40)$ VLM velocity profiles are still significantly less accurate and oscillate around the RGBEM velocity profiles. One observation to the credit of VLM, however, is that the mean (peak-to-peak averaged) velocity profiles appear to be better behaved near the plate edges; i.e., there are no "mean" overshoots similar to those seen in RGBEM profiles! This is most probably because the constant panel elements average out the singularity effects (due to the boundary edges) at the element centroids. This may suggest the possibility of using a hybrid RGBEM-VLM to handle the boundaries more robustly.

Figures $11 \mathrm{a}$ and $11 \mathrm{~b}$ represent the potential velocities evaluated by RGBEM in the parallel and normal directions to the flat plate, respectively, along $(0.5 \leq x, y \leq 1, z=0.1)$. We note that the profiles are smooth at this elevation, and that there are no signs of overshoot - once 
again suggesting that $[\Phi]$ must be represented more robustly at the edges. The VLM predictions at this elevation (not shown) are also smooth and fairly accurate.

The results presented thus far suggest that velocity profiles at evaluation points that are at least a (nominal) mesh size away from the body surface will be smooth. On the other hand, the profiles will "feel" the effect of the boundary for evaluation points that are within a (nominal) mesh size of the body surface. Unfortunately, since vorticity is concentrated in the thin boundary layer near the surface, the ability to evaluate smooth velocities near the boundary becomes a serious issue to contend with. For example, if during a computational timestep a vortex element repositions to a location which "feels" the spike in the normal velocity, it may quite possibly jump to the other side of the body - carrying with it the vorticity from the opposite side. One remedy, as we just alluded to, is to assign a very fine boundary element distribution so that the nominal mesh size is in the order of the boundary layer thickness. However, this is a highly inefficient proposition. A more robust alternative is to smooth out the discontinuity of the piecewise constant $\vec{n} \times \vec{\nabla}[\Phi]$ at the boundary element edges; i.e., to recreate linear elements with $C^{0}$ continuity of $\vec{n} \times \vec{\nabla}[\Phi]$ from piecewise constant solutions. To this end, one may apply areaweighted or simple arithmetic averaging techniques to assign to a vertex of a triangular element a value for $\vec{n} \times \vec{\nabla}[\Phi]$ that is the "average" of piecewise constant $\vec{n} \times \vec{\nabla}[\Phi]$ 's of all elements sharing that vertex. A linear variation of $\vec{n} \times \vec{\nabla}[\Phi]$ is then assumed using these averaged values at the vertices. As we will show shortly, the advantage of this approach is that the velocity profiles are smooth at arbitrarily close distances to the body surface. The disadvantage is that the (piecewise) solenoidality of $\vec{n} \times \vec{\nabla}[\Phi]$ is no longer imposed. At this point, we are unaware of robust smoothing algorithms that impose the solenoidality constraint.

Figures 12 demonstrate the effect of $\vec{n} \times \vec{\nabla}[\Phi]$ smoothing on the velocity profiles along $(0.5 \leq x, y \leq 1, z=0.001)$. The simple arithmetic averaging technique was used in this example. As expected, the jumps in the tangential velocity and the spikes in the normal velocity have altogether disappeared. This is despite the fact that the nominal mesh size (11x11 grids) is two orders of magnitude larger than the elevation $z=0.001$ of the evaluation points. Additionally, note that the normal velocity overshoot near the edge of the plate has dampened by an order of magnitude. Figures 13 depict the grid dependence of the "smooth" velocity profiles along $(0.5 \leq x, y \leq 1, z=0.001)$. We note that away from the edge, the velocities due to the low resolution simulation are acceptably close to the higher resolution results. However, near the edge, the magnitude of the tangential velocity increases with grid refinement - perhaps expectedly. Somewhat more peculiar is the behavior of the normal velocity near the edge where, while damped considerably compared to the unsmoothed case, the overshoots appear to be insensitive to grid refinement!

Next, we demonstrate qualitatively the application of RGBEM to geometries of interest to the parachute simulation. Figure 14a depicts the grid and $[\Phi]$ distributions on the surface of a hemispherical shell, the axis of symmetry of which is positioned along the free stream of unit speed. $[\Phi]$ is normalized by its peak value of -1.945 . Figure $14 \mathrm{~b}$ shows the velocity vector field proximal to the shell at its plane of symmetry. The velocity is normalized by the maximum value in the displayed field, 1.76. The shell surface in Fig. 14b is color coded by the potential jump 
distribution. Figures 15 are the corresponding results from the simulation of flow normal to the slotted hemisphere. The peak magnitudes of $[\Phi]$ on the surface and the fluid velocity are -0.348 and 1.97, respectively. Note that the grid resolution for this case is quite low; however, we are interested in the qualitative demonstration of the developed technology. 


\section{SUMMARY}

A Regularized Galerkin Boundary Element Method (RGBEM) was developed for simulating potential flow about zero thickness membranes. For collocation-based formulations, the boundary integral was shown to be singular unless the potential jump on the surface is at least $C^{1}$ continuous at the collocation points. RGBEM was formulated to relax the continuity constraint to $C^{0}$, and thus to eliminate the complexities involved with the development and implementation of methods based on the $C^{1}$ continuity restriction. The RGBEM formulation reduces the integrand singularity order by two via an a priori mathematical identification and removal of the singular components from the integral equation. This is in contrast to complex $a$ posteriori singularity removal strategies that are common in the literature. The new formulation was implemented assuming linear variation of the potential jump over planar triangular elements. This led to the double surface integration of the Green function only. The latter was evaluated analytically for the inner term and by an adaptive quadrature algorithm for the outer term. The exceptions to this were (1) the double surface integrals due to coincident elements and (2) the contributions to the integrals by the edges of edge-sharing elements; for which analytical expressions were derived. Finally, the superior accuracy of the linear RGBEM, as compared to the constant-patch Vortex Loop Method (VLM), was demonstrated using the test problem of potential flow normal to a flat square plate. 


\section{RECOMMENDATIONS}

The development of the generalized RGBEM formulation has provided the opportunity to evaluate the potential flow about thin and/or thick bodies at arbitrary orders of accuracy, without having to consider complex $C^{1}$ continuous elements and potential (jump) distributions. As demonstrated in Section 3, the accuracy of RGBEM predictions is far superior to those of the vortex loop method. Nevertheless, despite visible improvements, the velocity field proximal to the body surface contains jumps and spikes, which grow as the evaluation points approach the collocation points on the surface. This is because the potential (jump) is $C^{0}$ continuous. In Section 3, we demonstrated a simple smoothing remedy at the post-processing stage to render the tangential gradients of the potential (jump) $C^{0}$ continuous. The proposed smoothing technique performed remarkably well, at least for the simple case of flow normal to a flat plate. However, the primary disadvantage of this approach is that the tangential gradients of the potential (jump) are no longer solenoidal - not even piecewise! For this purpose, more advanced smoothing techniques, which account for curvature effects at the collocation points as well as the solenoidality of the potential jump gradients, need to be developed in the near future. One possibility may be the development of Galerkin smoothing techniques for the tangential gradients quite similar to the one developed in this report [14].

The processes of matrix assembly and field point evaluation in RGBEM are relatively time consuming, which may well become the bottleneck in the vortex-boundary element simulation stage. A fast parallel multipole capability must be developed for RGBEM to reduce the complexity of the computations and storage requirements to manageable levels. In addition, for non-contiguous boundary elements that are at least a nominal mesh size away from each other, the use of a degree 13 adaptive quadrature scheme to evaluate the integrals to machine precision may be quite unnecessary. The best strategy is to apply quadratures of varying orders, depending on the relative position of the two elements involved in the integration.

The exact cause for the observed overshoot in the normal velocity field proximal to the edge singularity remains unresolved. It is quite possible that the implementation of special boundary elements, similar to the ones applied in crack propagation analyses, will alleviate this difficulty. Another approach may be to "smooth out" the edge singularity; i.e., to assign an arbitrarily small curvature to the edge which can be accounted for analytically. 


\section{REFERENCES}

1 J. L. Hess and A. M. O. Smith, "Calculation of Non-Lifting Potential Flow About Arbitrary Three-Dimensional Bodies," Douglas Aircraft Company Report No. ES 40622, 1962.

2 B. Maskew, "Program VSAERO Theory Document: A Computer Program for Calculating Nonlinear Aerodynamic Characteristics of Arbitrary Configurations," NASA CR-4023, 1987.

3 J. E. Romate, "Local Error Analysis of Three-Dimensional Panel Methods In Terms of Curvilinear Surface Coordinates," SIAM J. Numer. Anal., Vol. 27, No. 2, pp. 529-542, 1990.

4 C. Balakrishna, L. J. Gray and J. H. Kane, "Efficient Analytical Integration of Symmetric Galerkin Boundary Integrals Over Curved Elements: Thermal Conduction Formulation," Comp. Meth. Appl. Mech. Engr., Vol. 111, pp. 335-355, 1994.

5 P. Parreira and M. Guiggiani, "On the Implementation of the Galerkin Approach In the Boundary Element Method," Fluids \& Structures, Vol. 33, No. 1, pp. 269-279, 1989.

6 H. Y. Lamb, Hydrodynamics, Dover Publications, N. Y., 1945.

7 R. I. Lewis, Vortex Element Methods for Fluid Dynamic Analysis of Engineering Systems, Cambridge Univ. Press, 1991.

8 A. Gharakhani and A. F. Ghoniem, "BEM Solution of the 3D Internal Neumann Problem And A Regularized Formulation For the Potential Velocity Gradients," Int. J. Numer. Meth. Fluids, Vol. 24, pp. 81-100, 1997.

9 D. E. Medina and J. A. Liggett, "Three Dimensional Boundary Element Computation of Potential Flow In Fractured Rock," Int. J. Numer. Meth. Eng., Vol. 26, pp. 2319-2330, 1988.

10 Algorithm 706 Source Code, http://www.netlib.org/toms/706.

$11 \mathrm{~J}$. Berntsen and T. O. Espelid, "Algorithm 706: DCUTRI: An Algorithm For Adaptive Cubature Over A Collection of Triangles," ACM Trans. Math. Software, Vol. 18, No. 3, pp. 329-342, 1992.

12 J. Berntsen and T. O. Espelid, "Degree 13 Symmetric Quadrature Rules For the Triangle," Reports In Informatics No. 44, Dept. of Informatics, Univ. of Bergen, Norway, 1990.

13 L. J. Gray, Private Communications, Oak Ridge National Laboratories, 1998.

14 L. J. Gray, D. Maroudas and M. N. Enmark, "Galerkin Boundary Integral Method for Evaluating Surface Derivatives," Comp. Mech., Vol. 22, pp. 187-193, 1998.

15 L. M. Milne-Thomson, Theoretical Hydrodynamics, Dover Publications, N. Y., 1996. 


\section{FIGURES}

Figure 1: Schematic depiction of a thin body in free stream.

Figure 2: Schematic diagram of the coordinate system local to a boundary element.

Figure 3: Schematic diagram depicting (1) the panel-panel interaction in the Galerkin method and (2) the local coordinate system used in the Galerkin integrations.

Figures 4 - 13: Potential flow normal to a flat square plate

Figure 4: Grid dependence study of (a) RGBEM and (b) VLM predictions of the surface potential jump along the symmetry line of the plate.

Figure 5: VLM prediction of the potential velocity vector field in the vicinity (and in the plane of symmetry) of the plate.

Figure 6: Grid dependence study of the (a) tangential and (b) normal velocity predictions by RGBEM - at 0.001 units above the plate.

Figure 7: Grid dependence study of the (a) tangential and (b) normal velocity predictions by VLM - at 0.001 units above the plate.

Figure 8: A comparison between the RGBEM and VLM predictions of the (a) tangential and (b) normal velocity components at 0.001 units above the plate.

Figure 9: Grid dependence study of the (a) tangential and (b) normal velocity predictions by RGBEM - at 0.01 units above the plate.

Figure 10: A comparison between the RGBEM and VLM predictions of the (a) tangential and (b) normal velocity components at 0.01 units above the plate.

Figure 11: Grid dependence study of the (a) tangential and (b) normal velocity predictions by RGBEM - at 0.1 units above the plate.

Figure 12: Effect of surface velocity smoothing on the smoothness of the (a) tangential and (b) normal velocity predictions by RGBEM - at 0.001 units above the plate.

Figure 13: Grid dependence study of the (a) tangential and (b) normal velocity predictions by the "smoothed" RGBEM - at 0.001 units above the plate.

Figure 14: Potential flow normal to (and along the axis of symmetry of) a hemispherical shell: (a) Surface grid and potential jump distributions. (b) Velocity vector field proximal to the shell and at its plane of symmetry. 
Figure 15: Potential flow normal to (and along the axis of symmetry of) a slotted hemispherical shell: (a) Surface grid and potential jump distributions. (b) Velocity vector field proximal to the shell and at its plane of symmetry.

Figure B-1: Blown up schematic of an arbitrarily small boundary surface in the vicinity of a collocation point. 

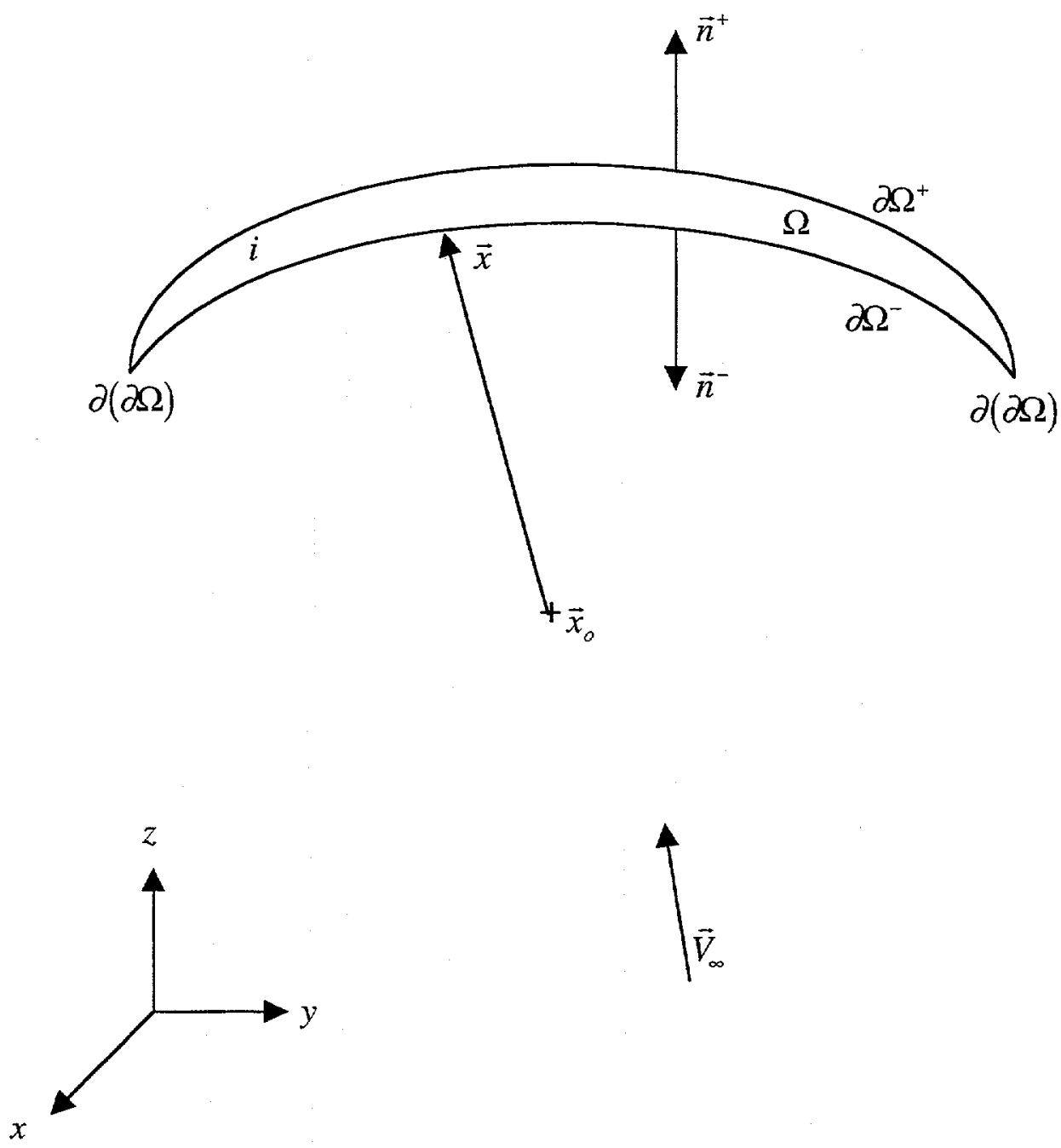

$\hat{V_{\infty}}$

Figure 1 

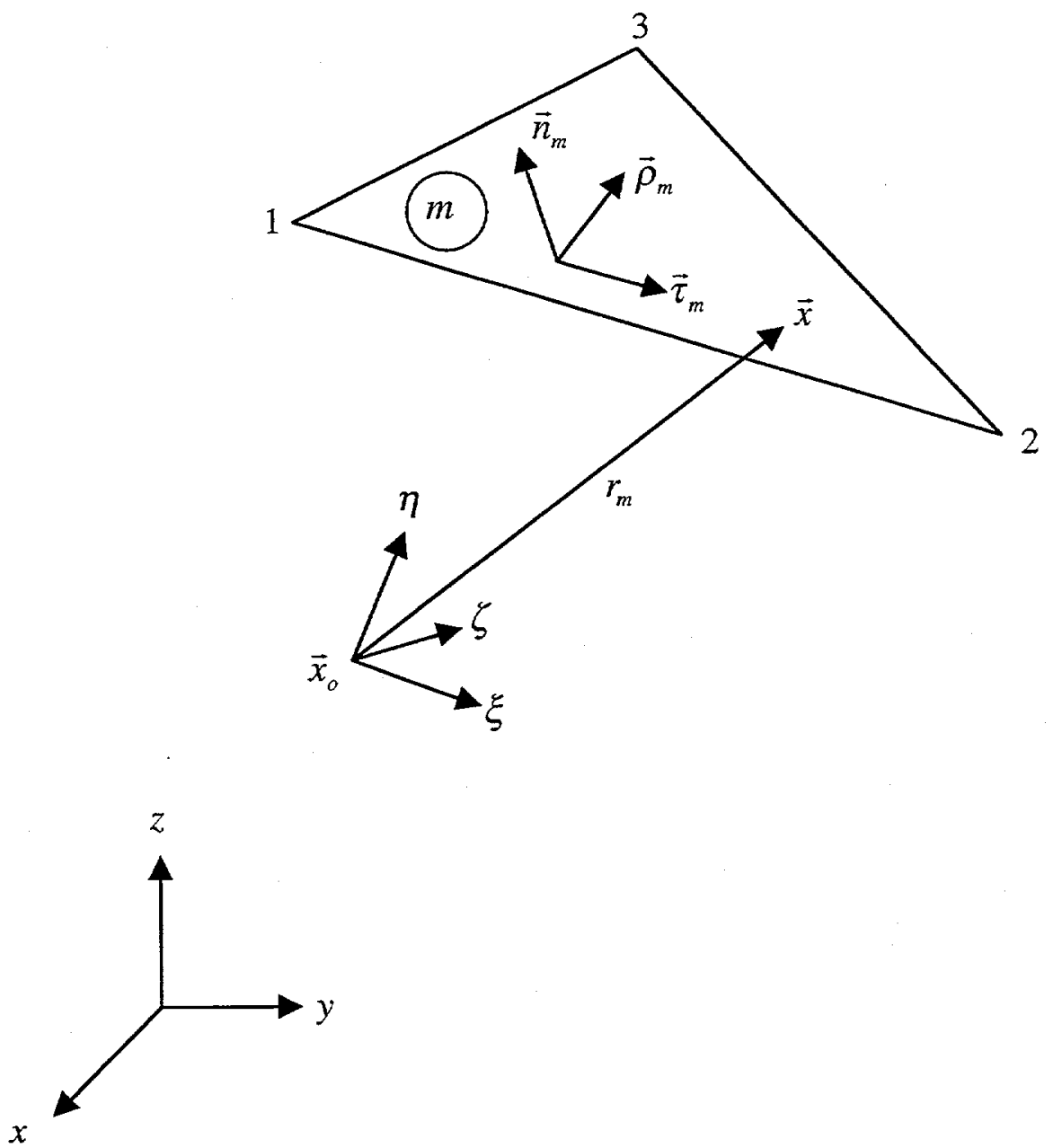

Figure 2 

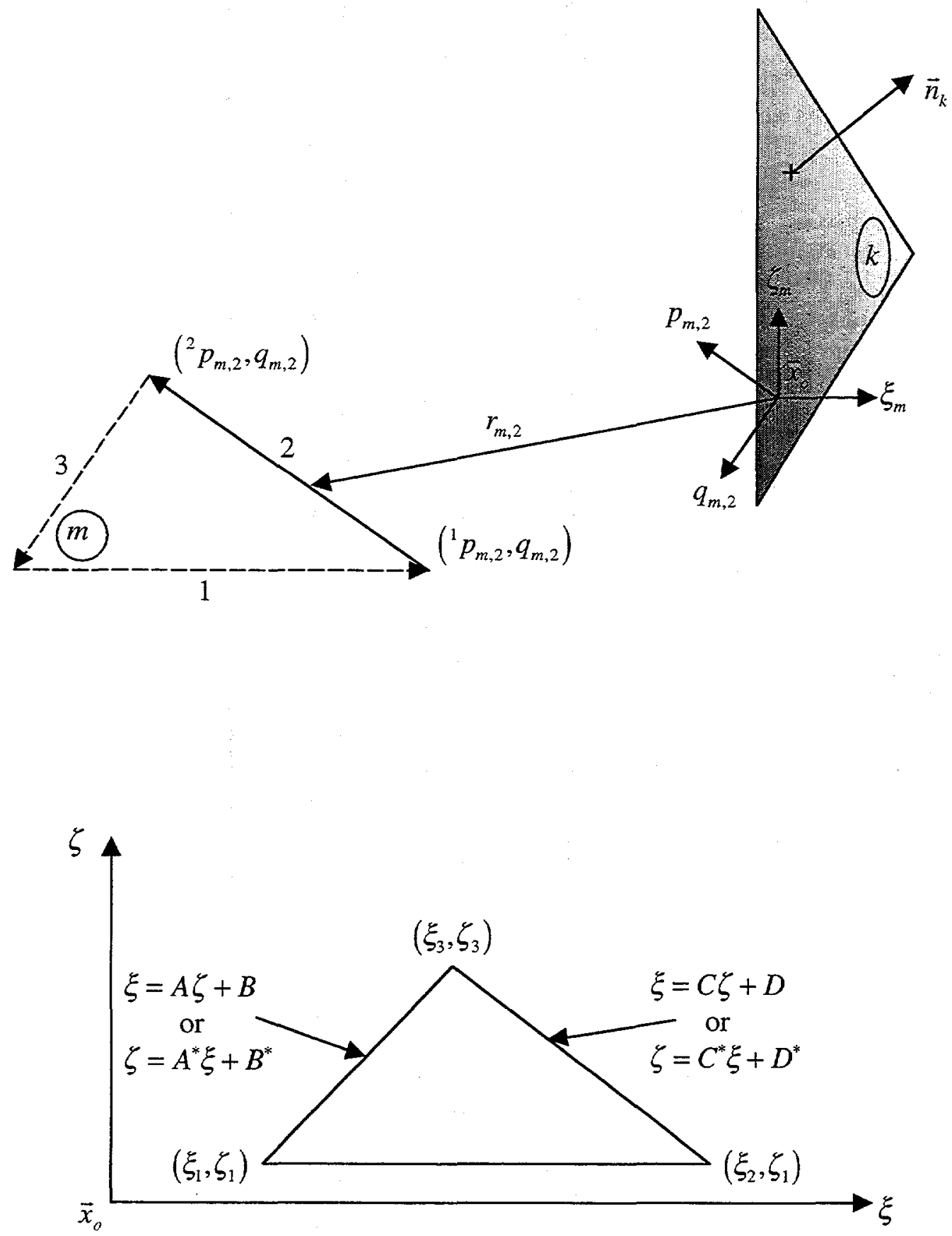

Figure 3 


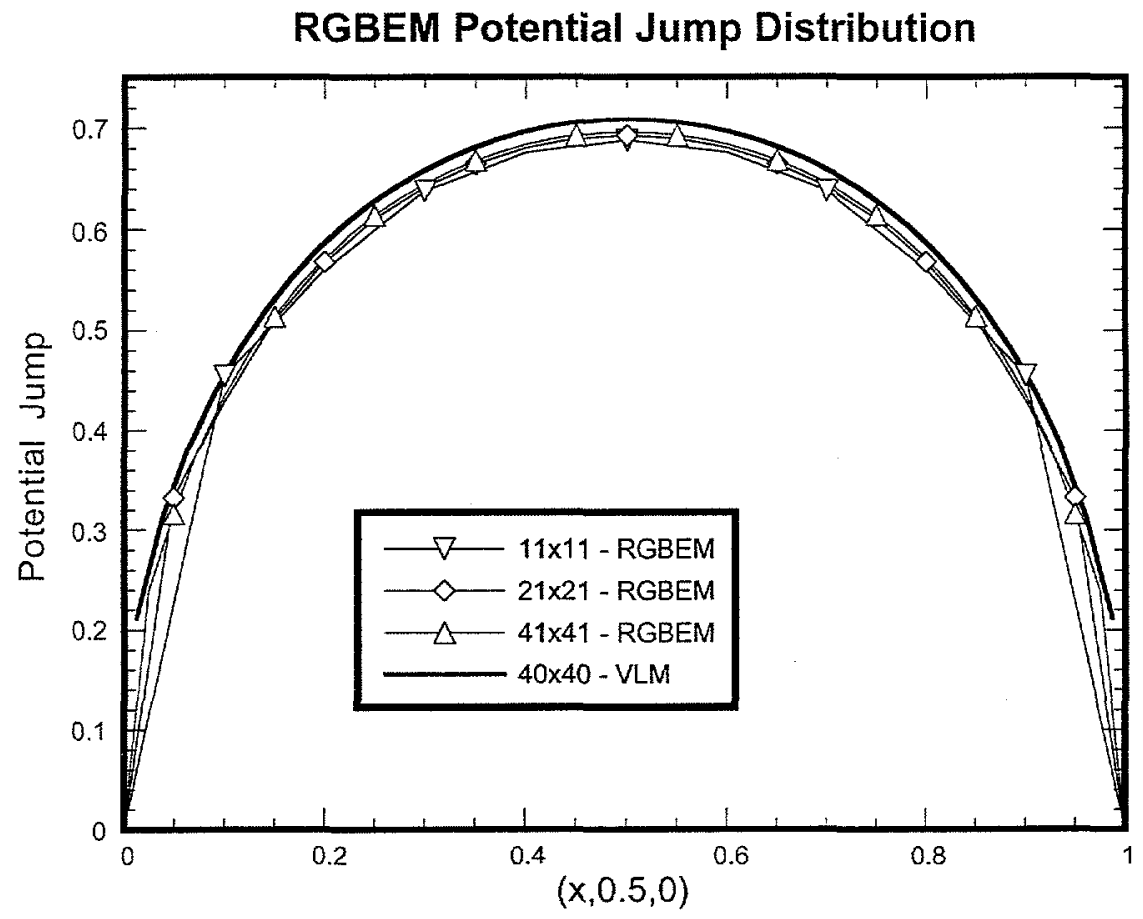

(a)

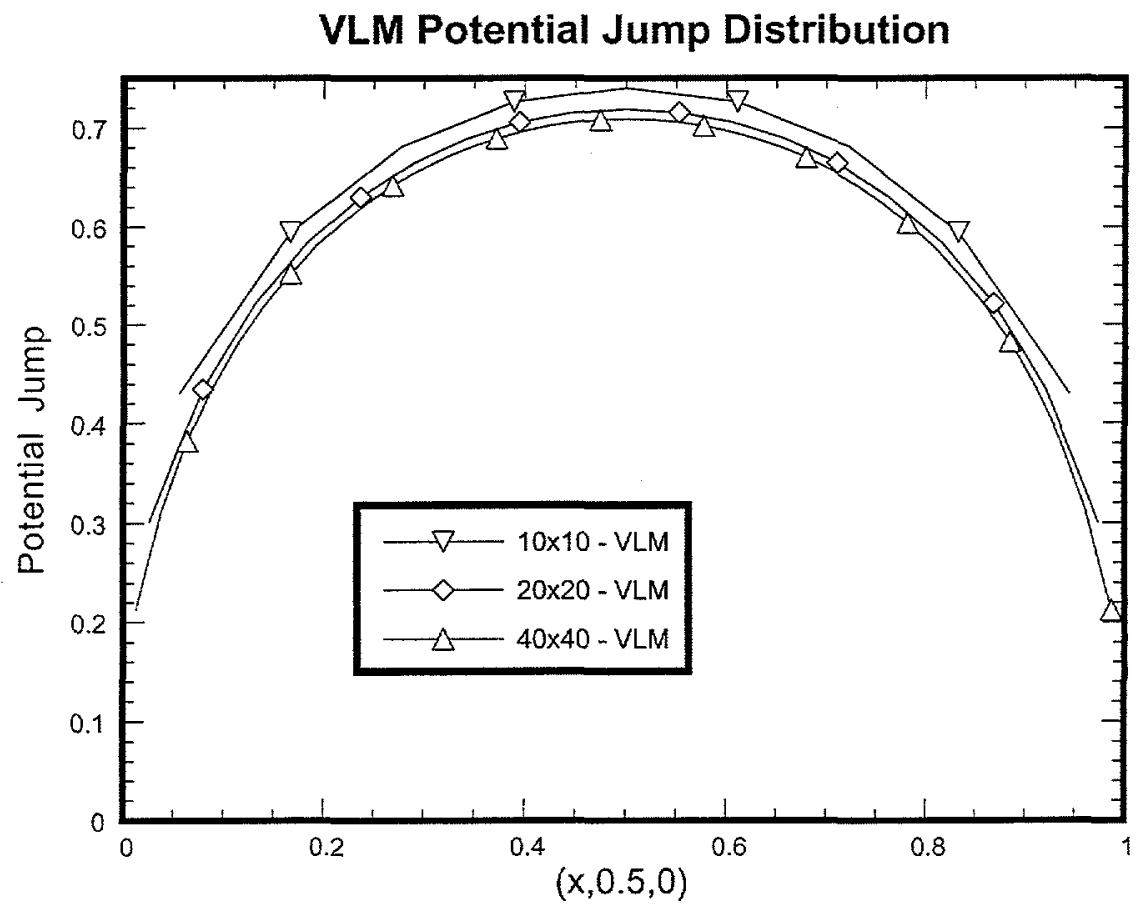

(b)

Figure 4 


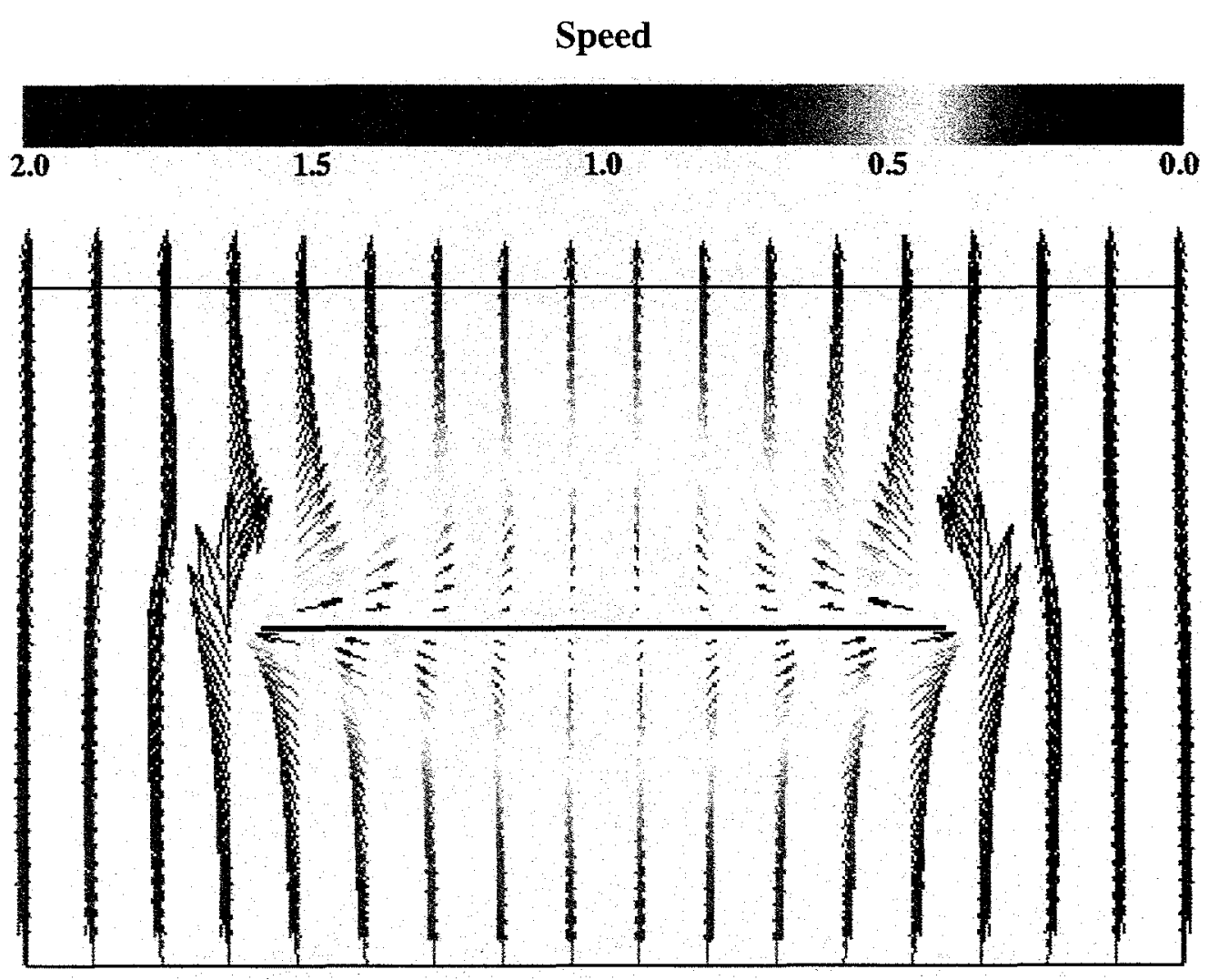

Figure 5 
Grid Dependence of RGBEM Predictions At $\mathrm{z}=\mathbf{0 . 0 0 1}$

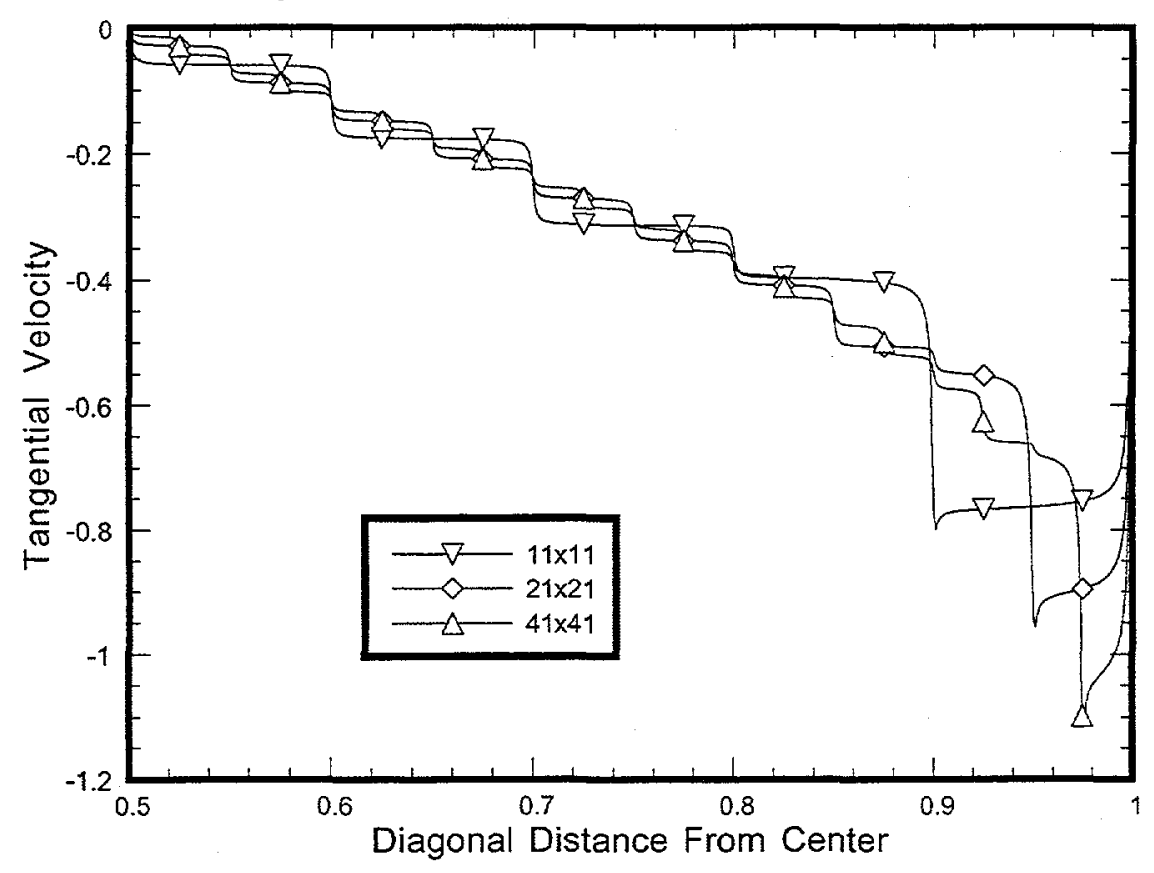

(a)

Grid Dependence of RGBEM Predictions At $\mathbf{z}=\mathbf{0 . 0 0 1}$

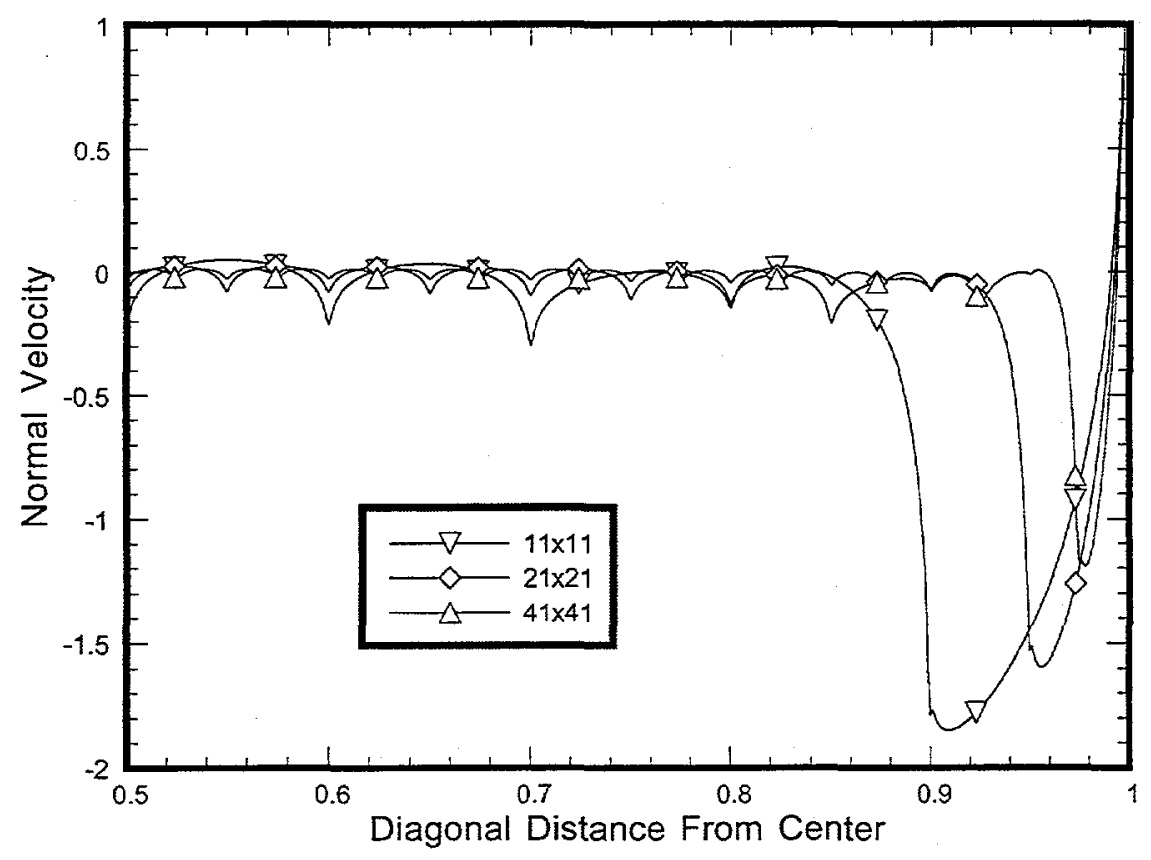

(b)

Figure 6 


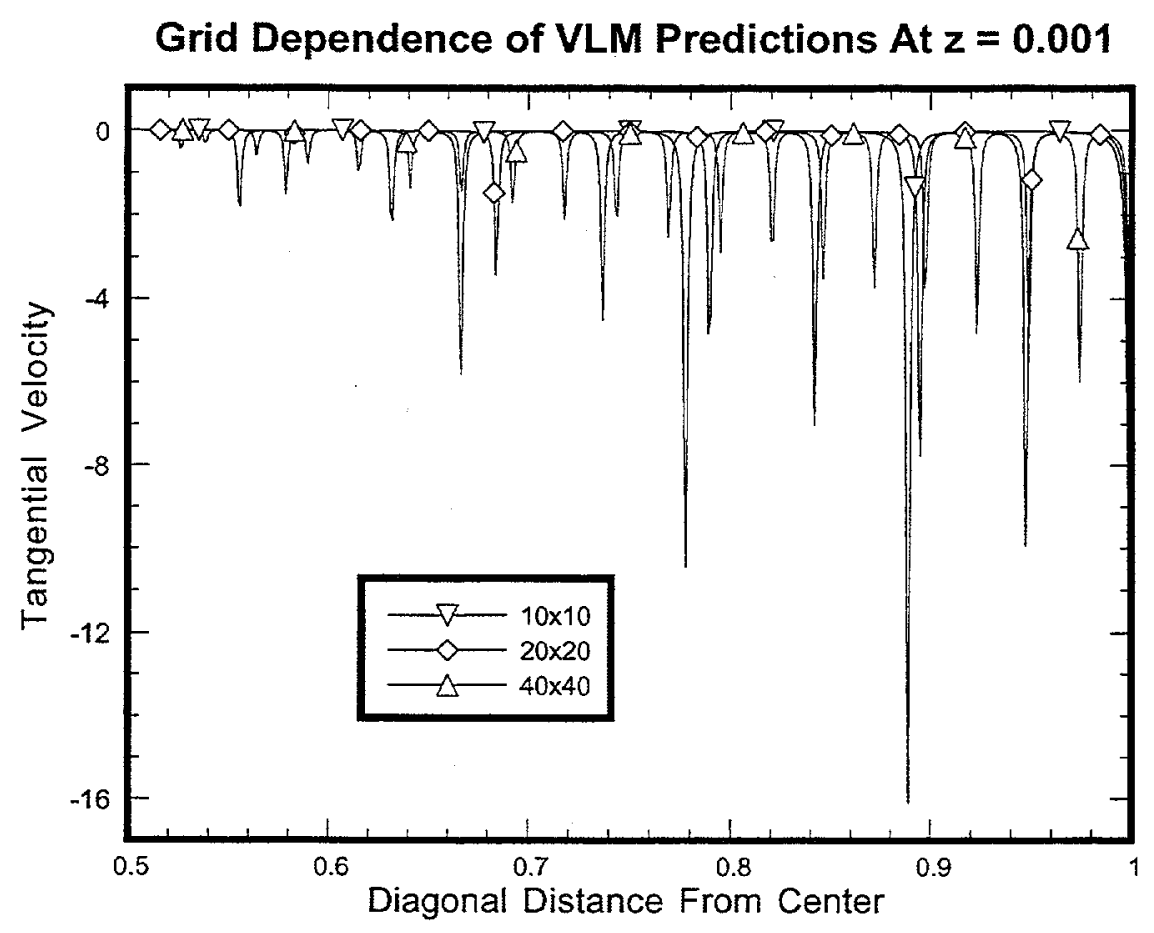

(a)

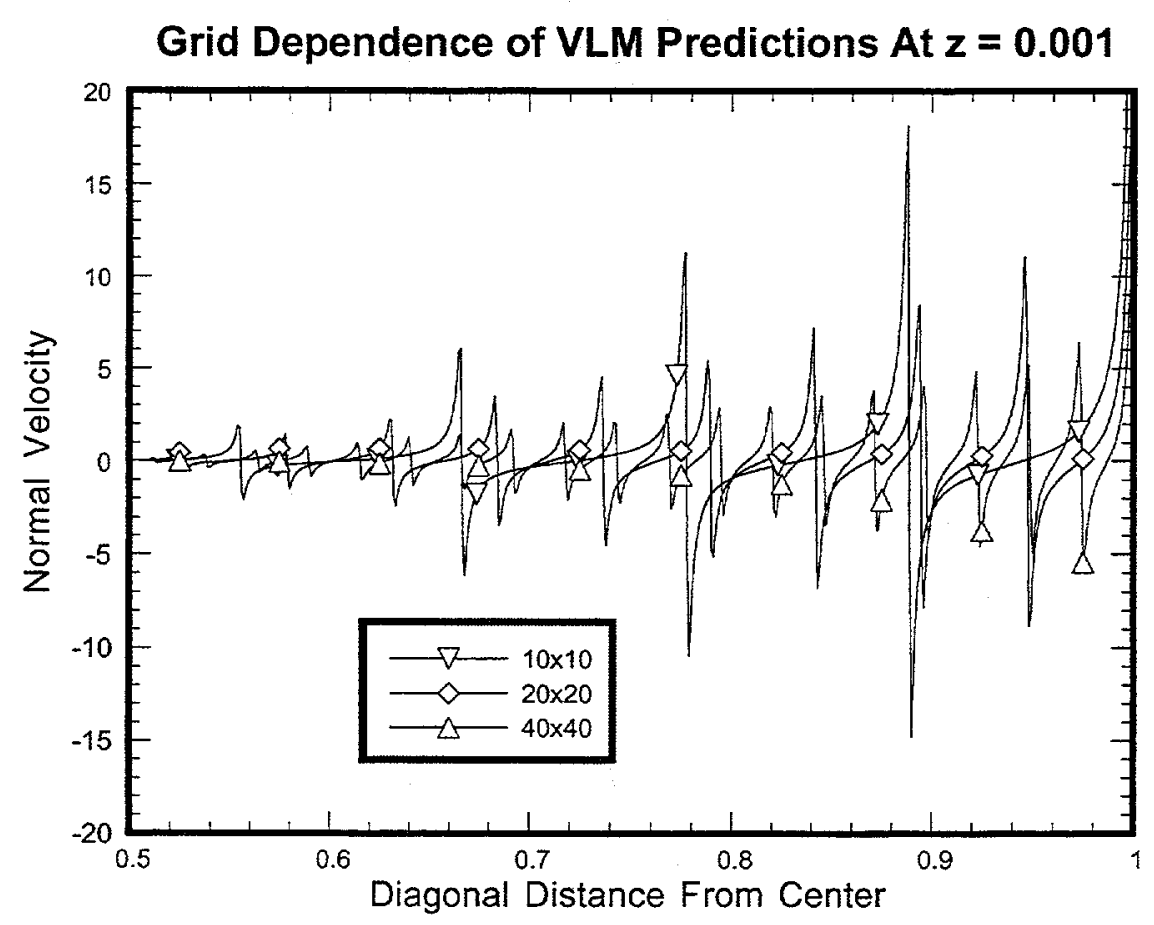

(b)

Figure 7 


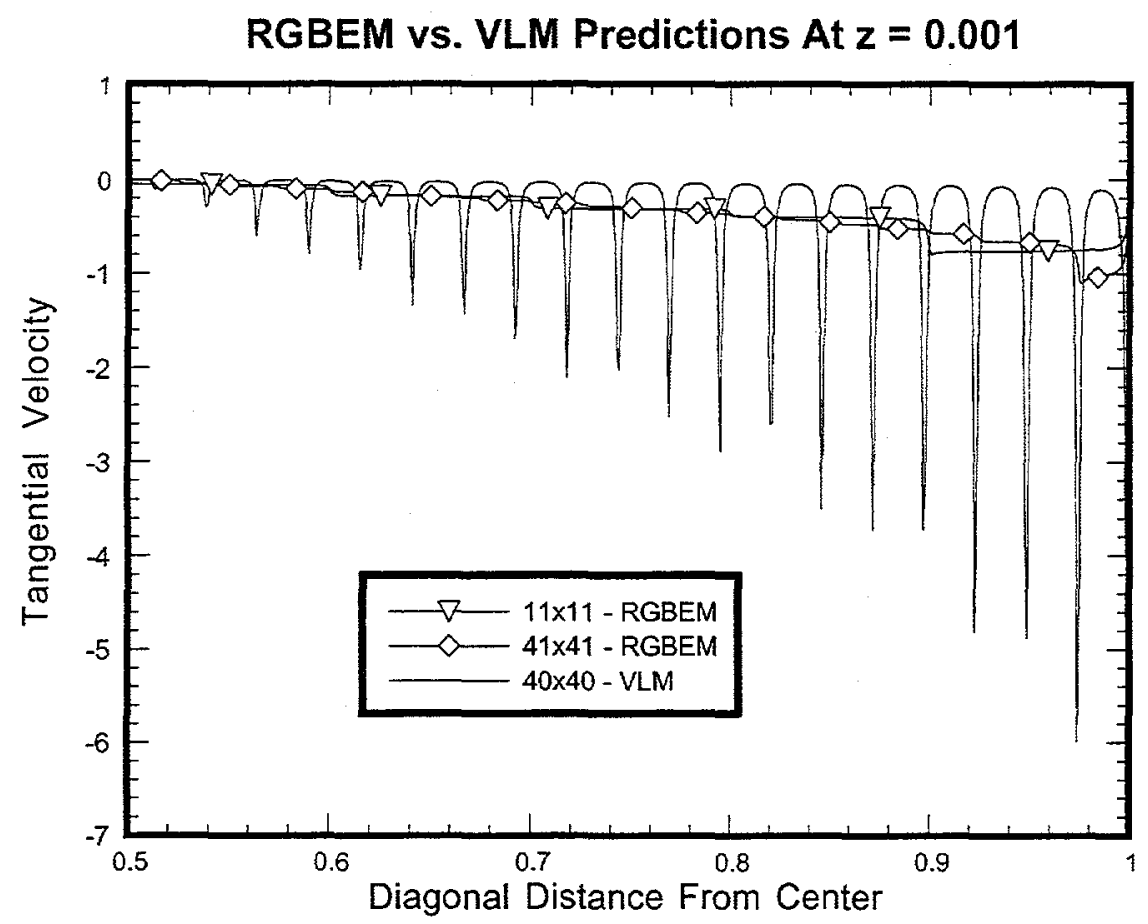

(a)

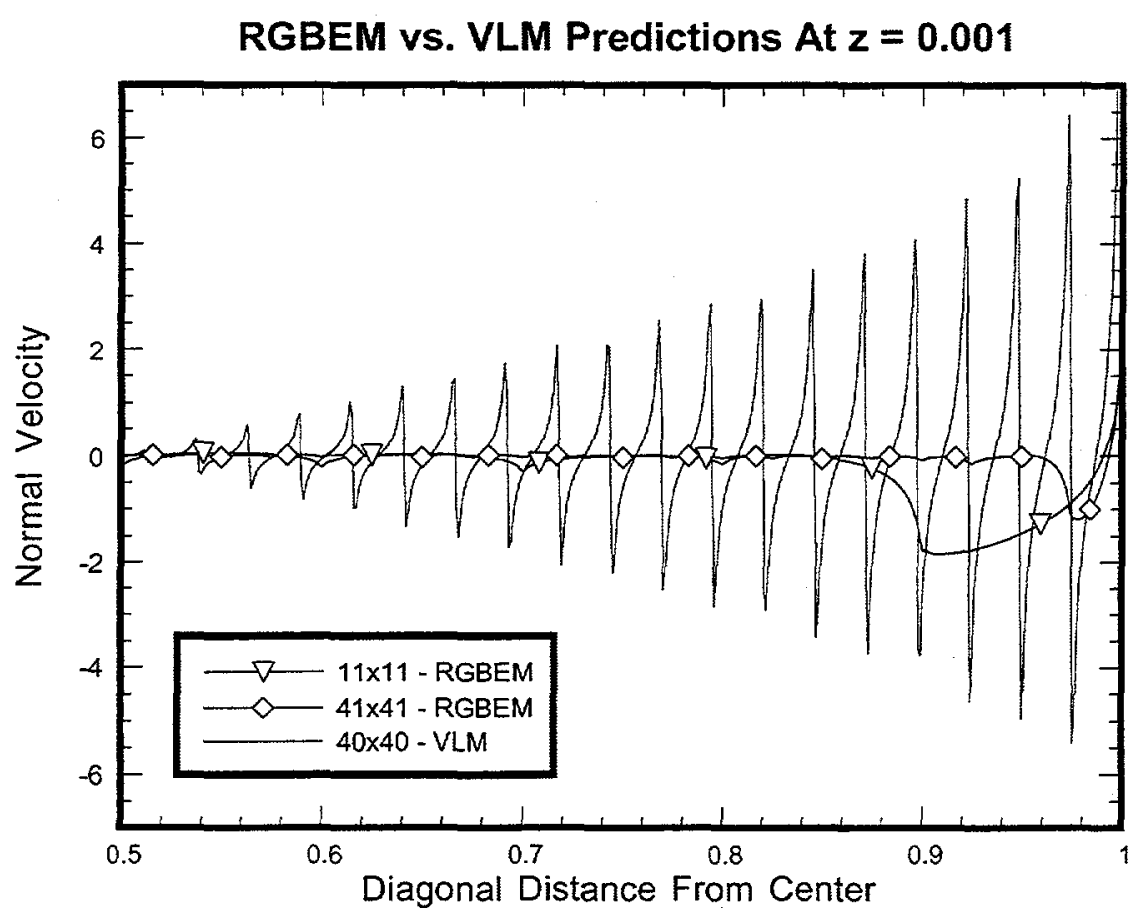

(b)

Figure 8 
Grid Dependence of RGBEM Predictions At $\mathbf{z}=0.01$

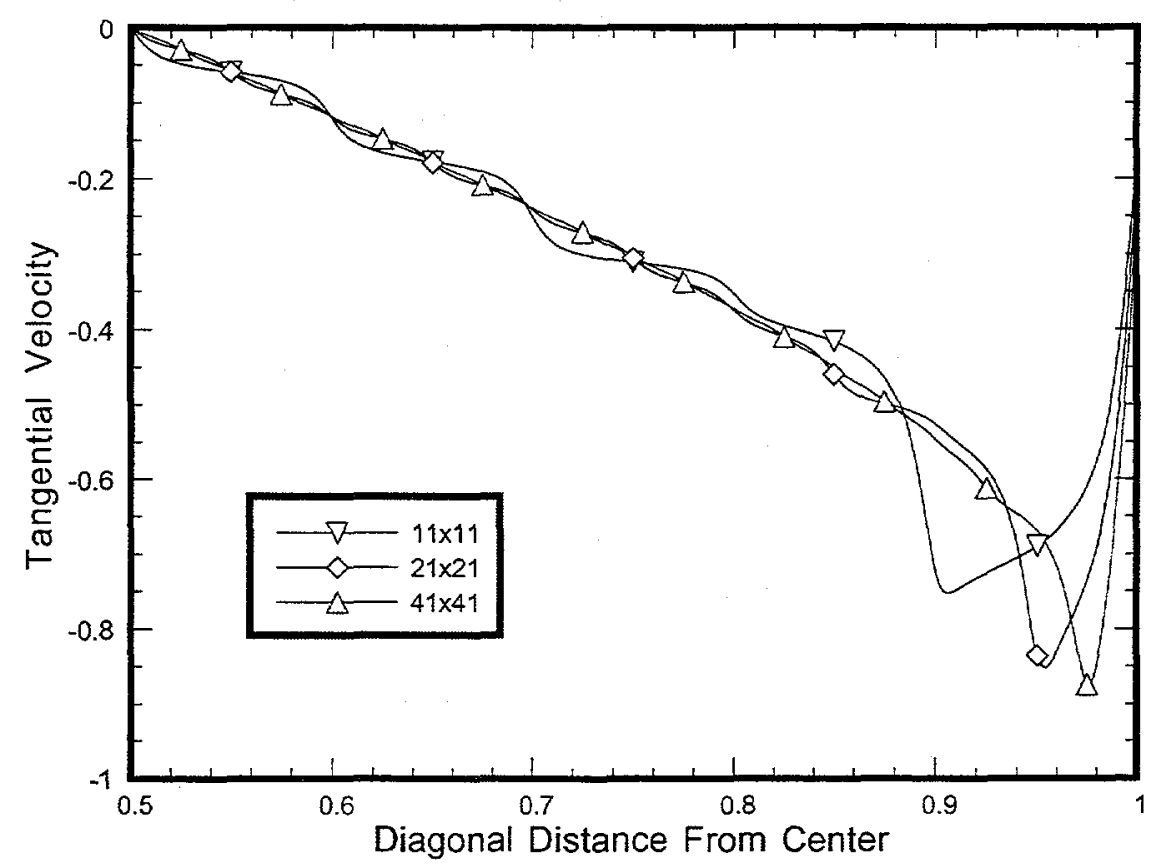

(a)

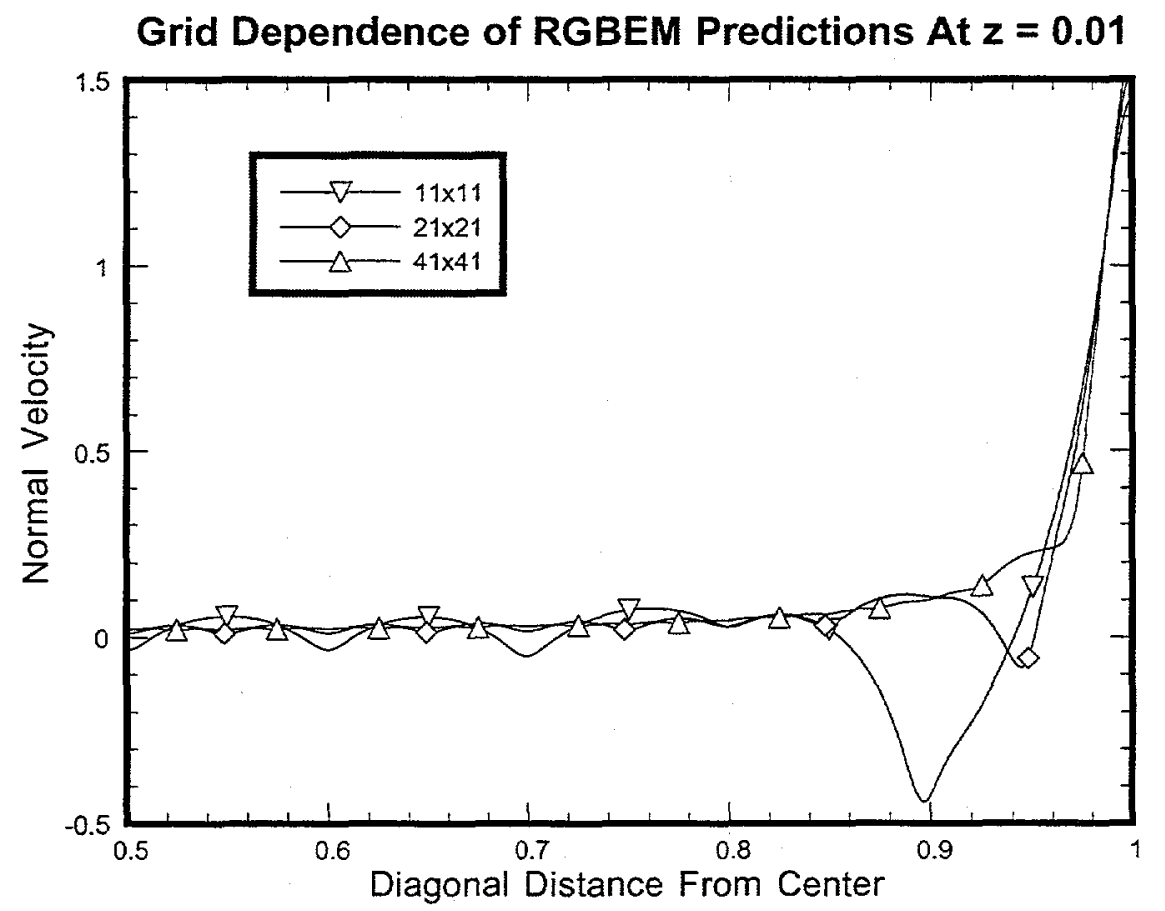

(b)

Figure 9 
RGBEM vs. VLM Predictions At $z=0.01$

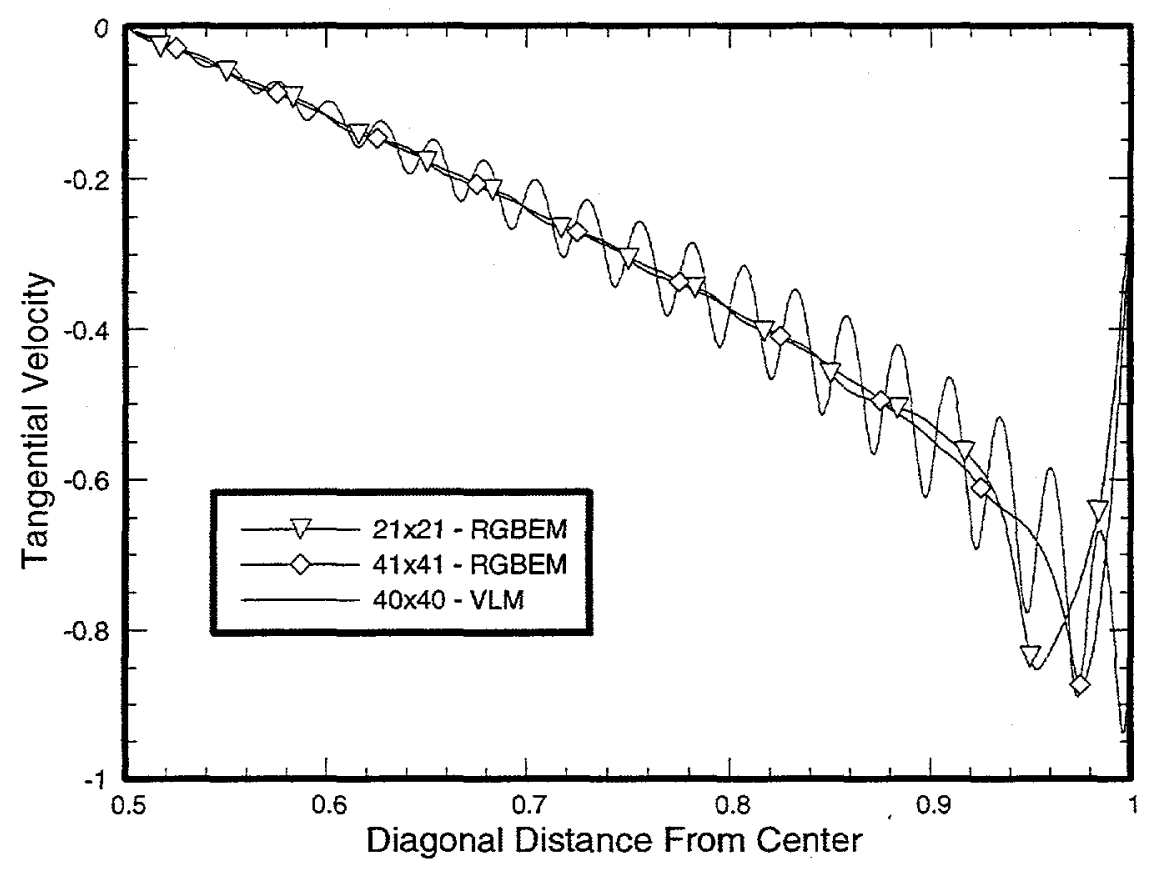

(a)

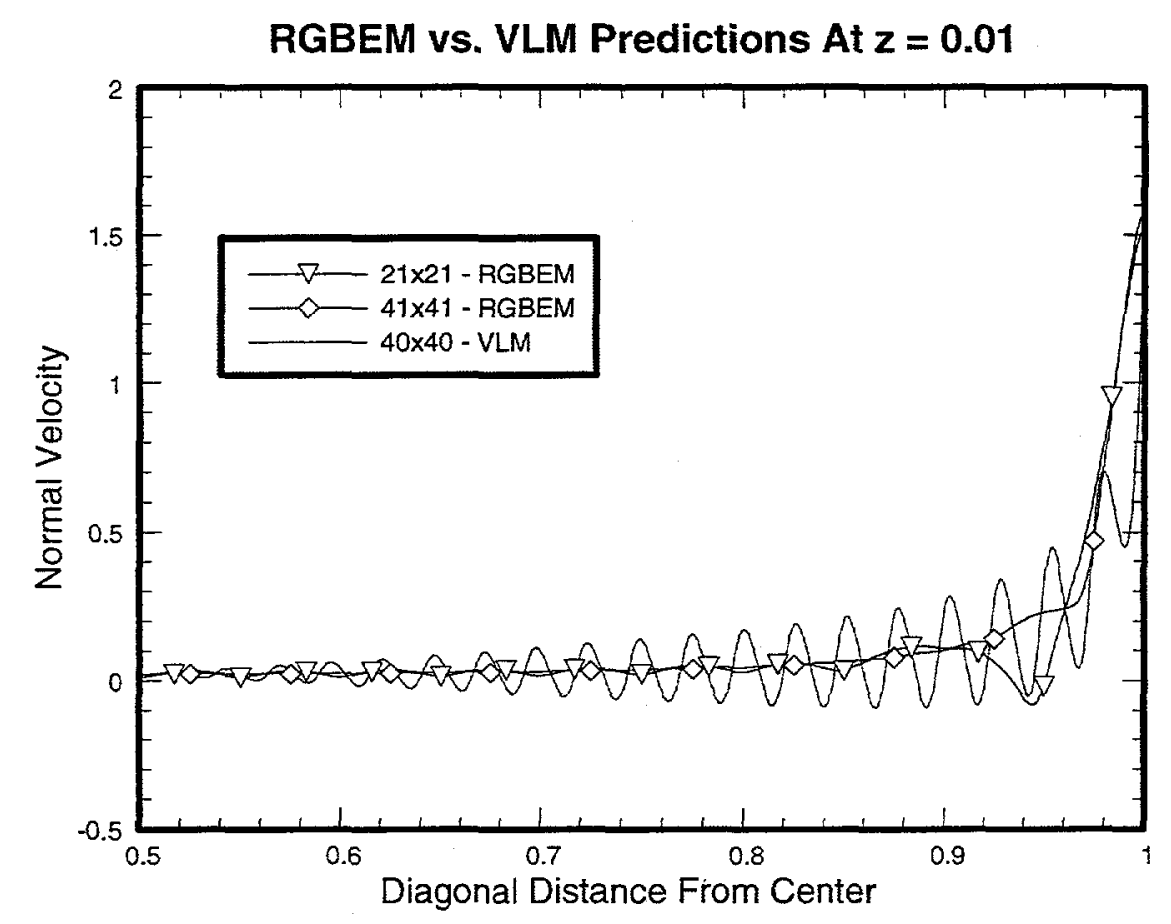

(b)

Figure 10 


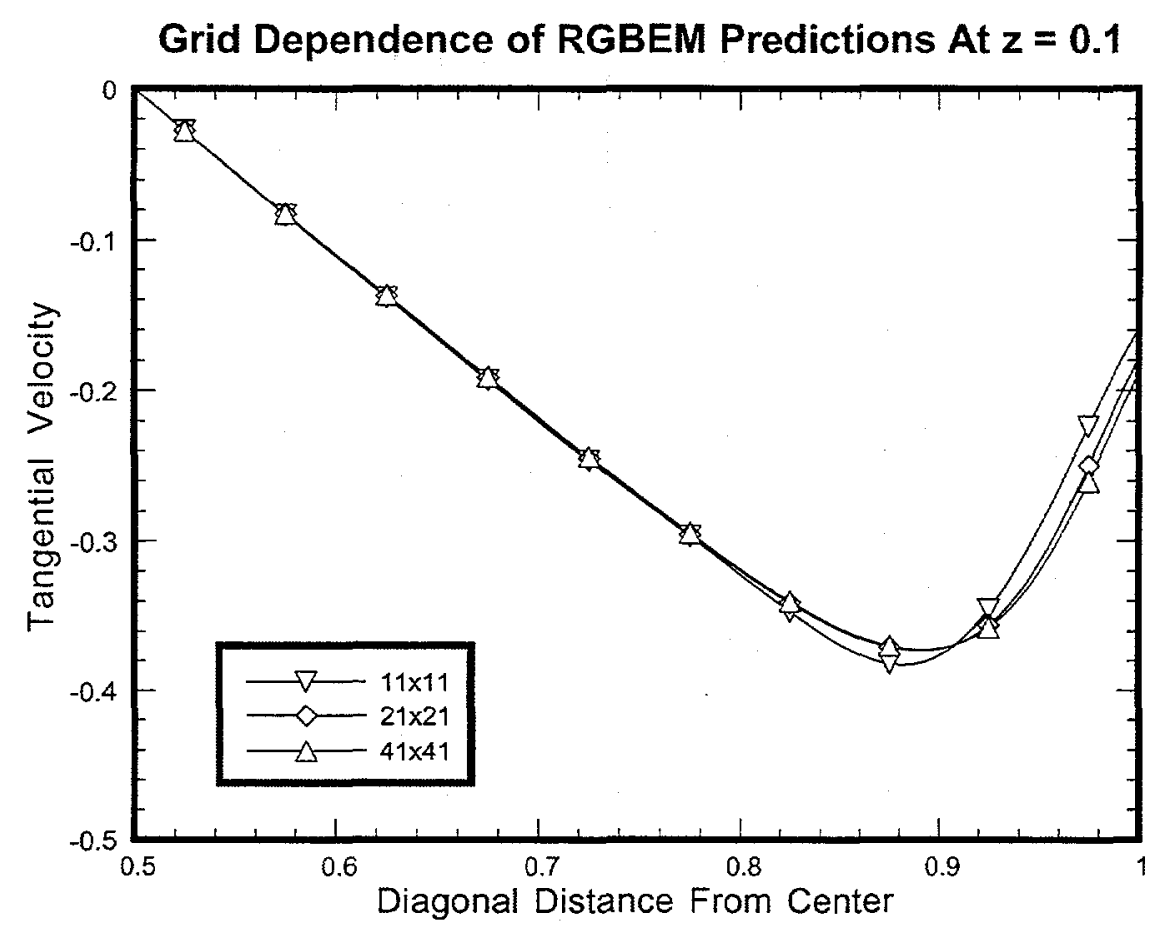

(a)

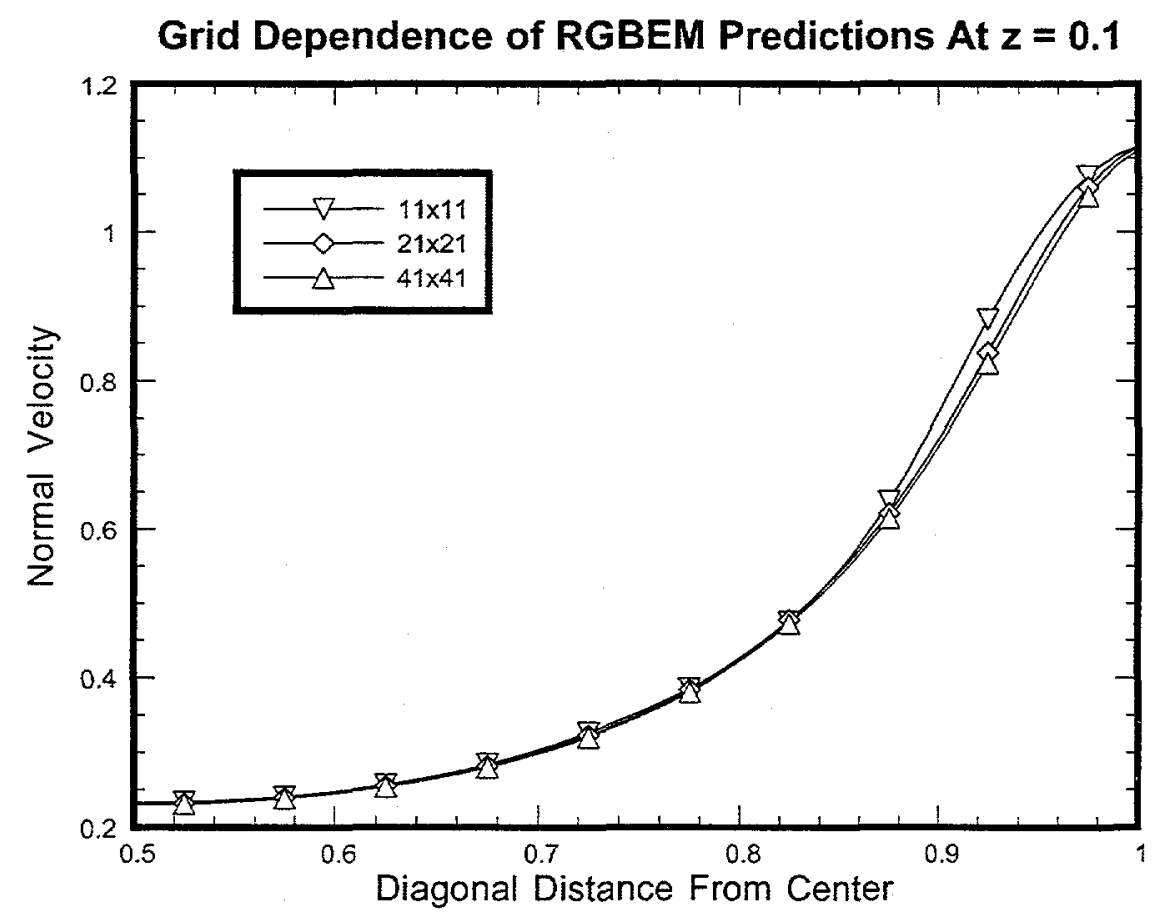

(b)

Figure 11 
Effect of Smoothing On RGBEM Predictions At $z=0.001$

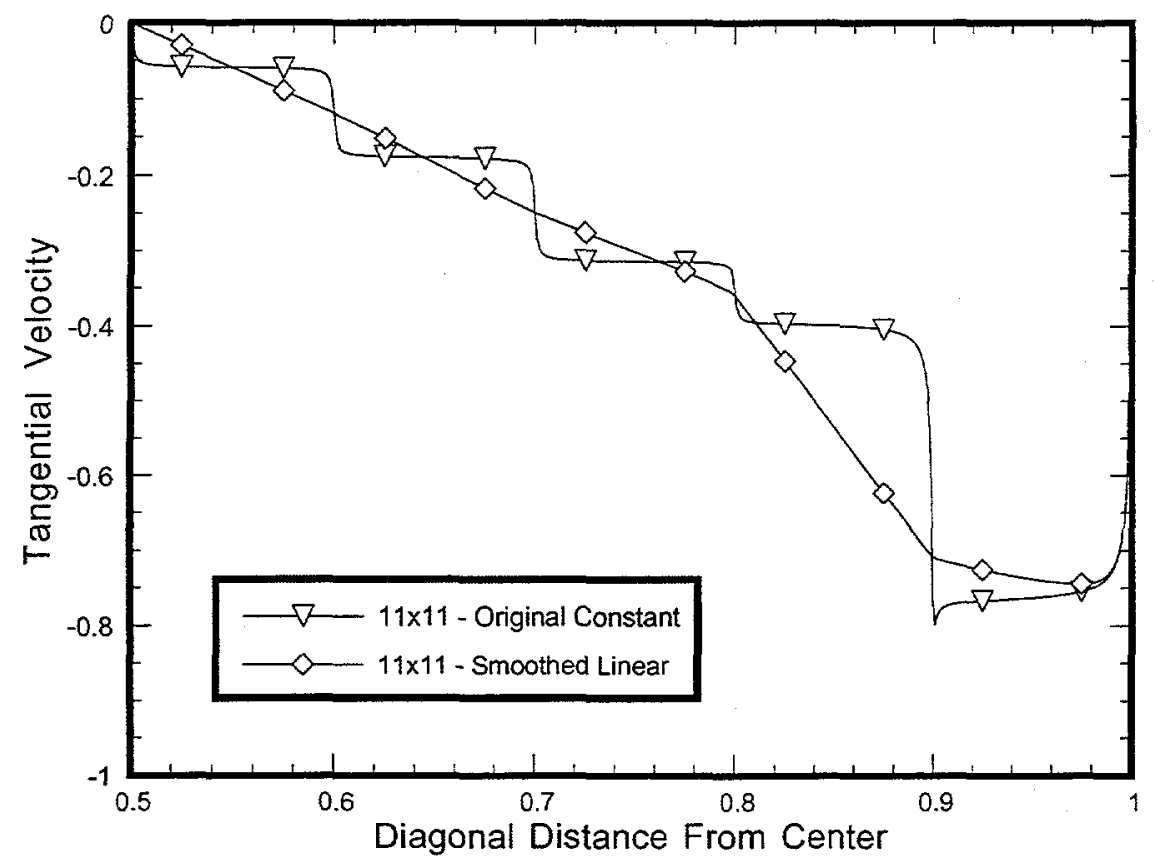

(a)

Effect of Smoothing On RGBEM Predictions At $z=0.001$

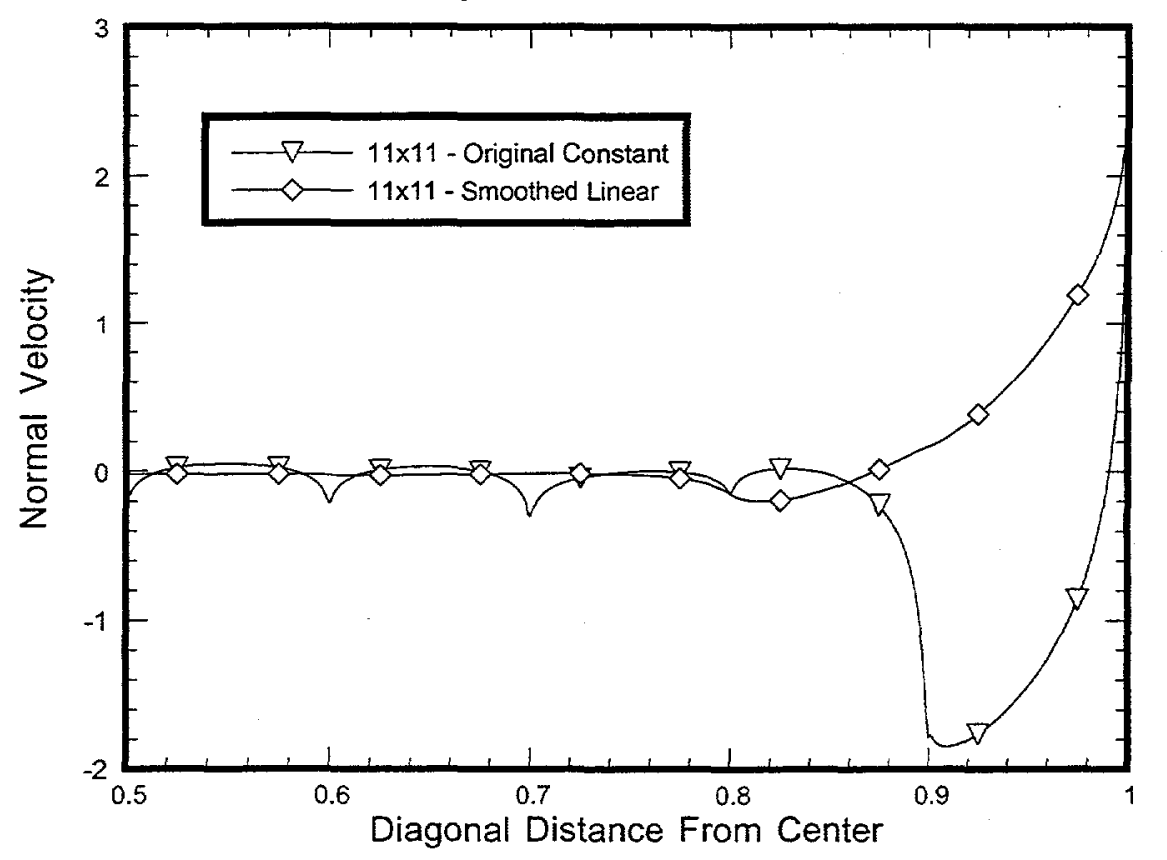

(b)

Figure 12 
Grid Dependence of Smoothed RGBEM Predictions At $z=0.001$

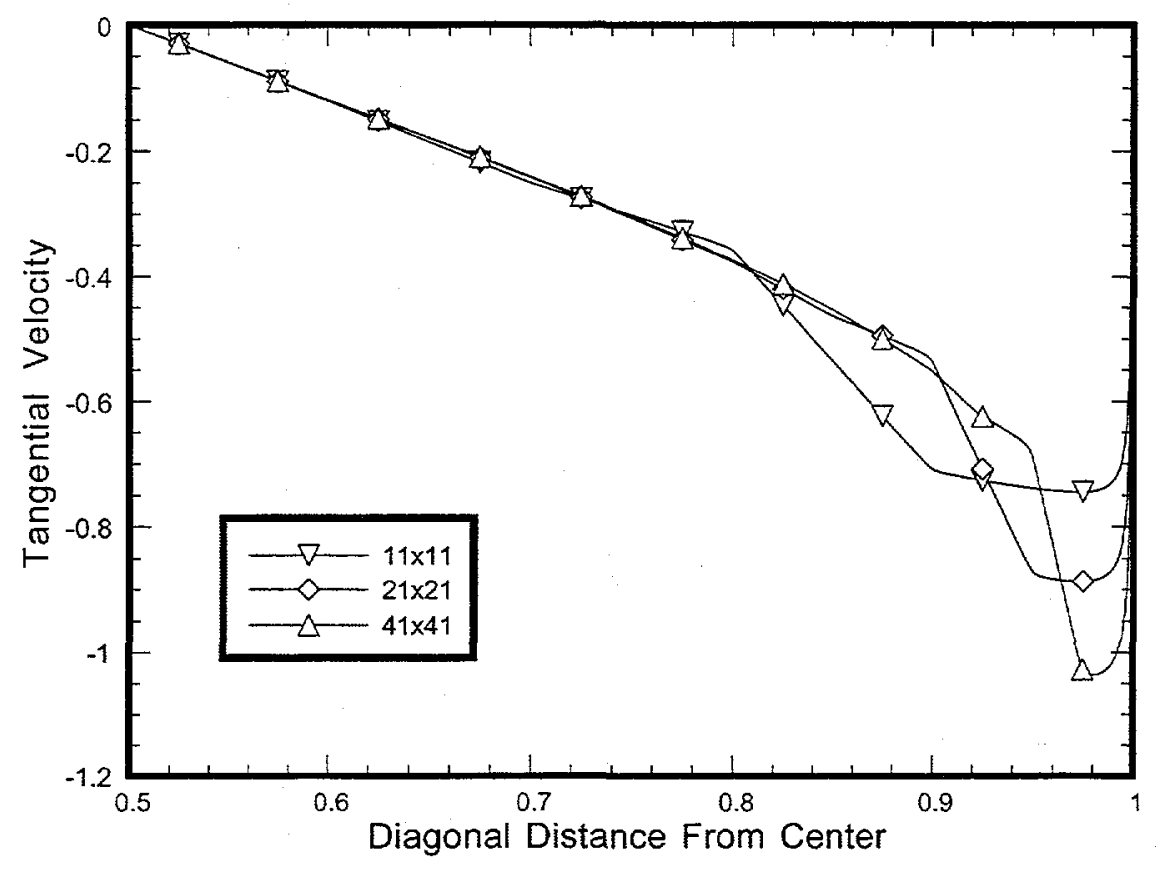

(a)

Grid Dependence of Smoothed RGBEM Predictions At $z=0.001$

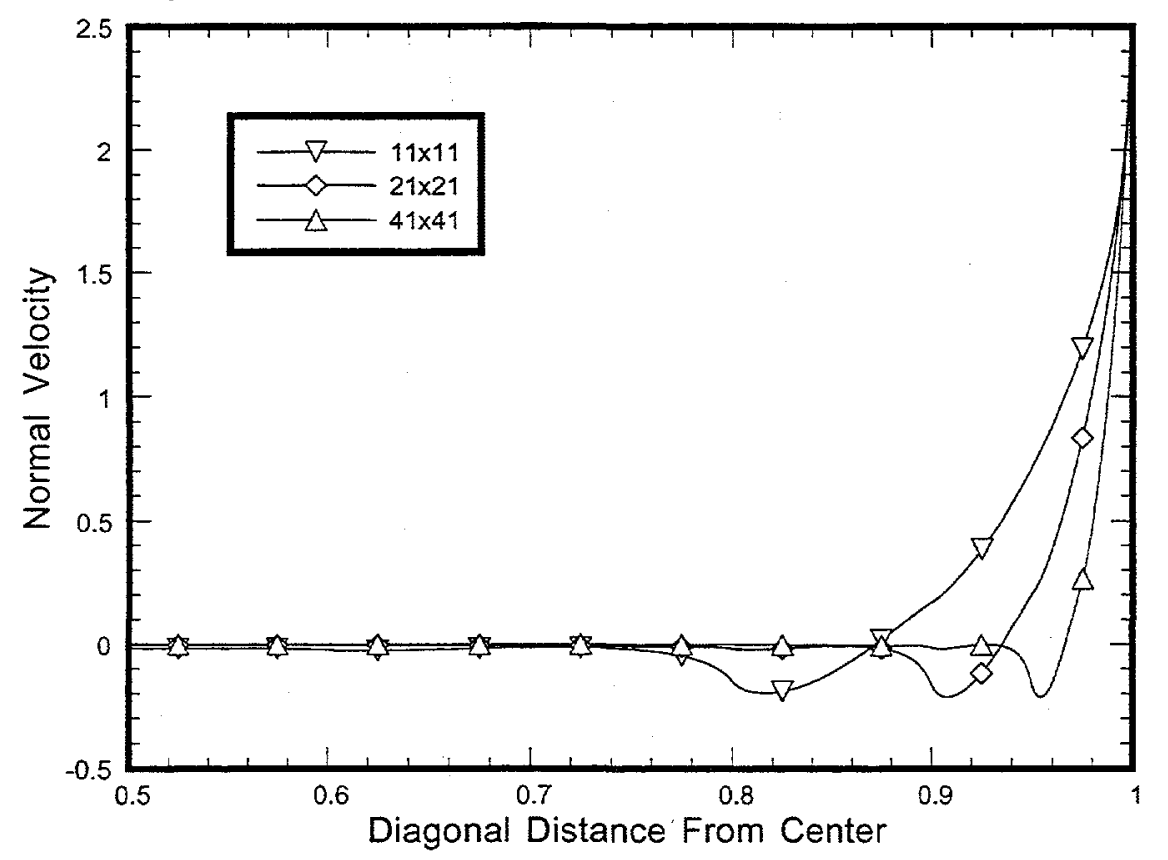

(b)

Figure 13 


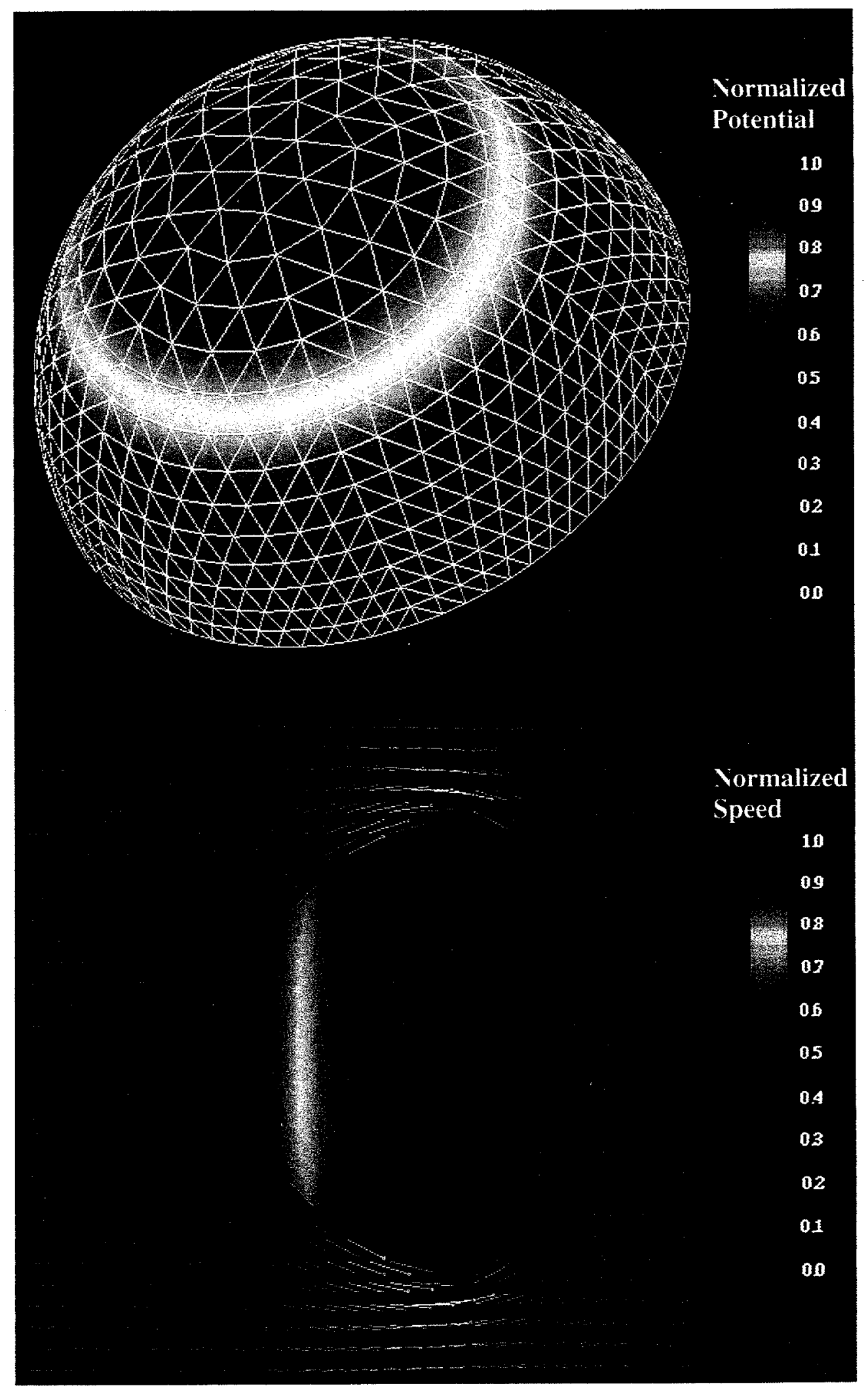

(a)

(b)

Figure 14 


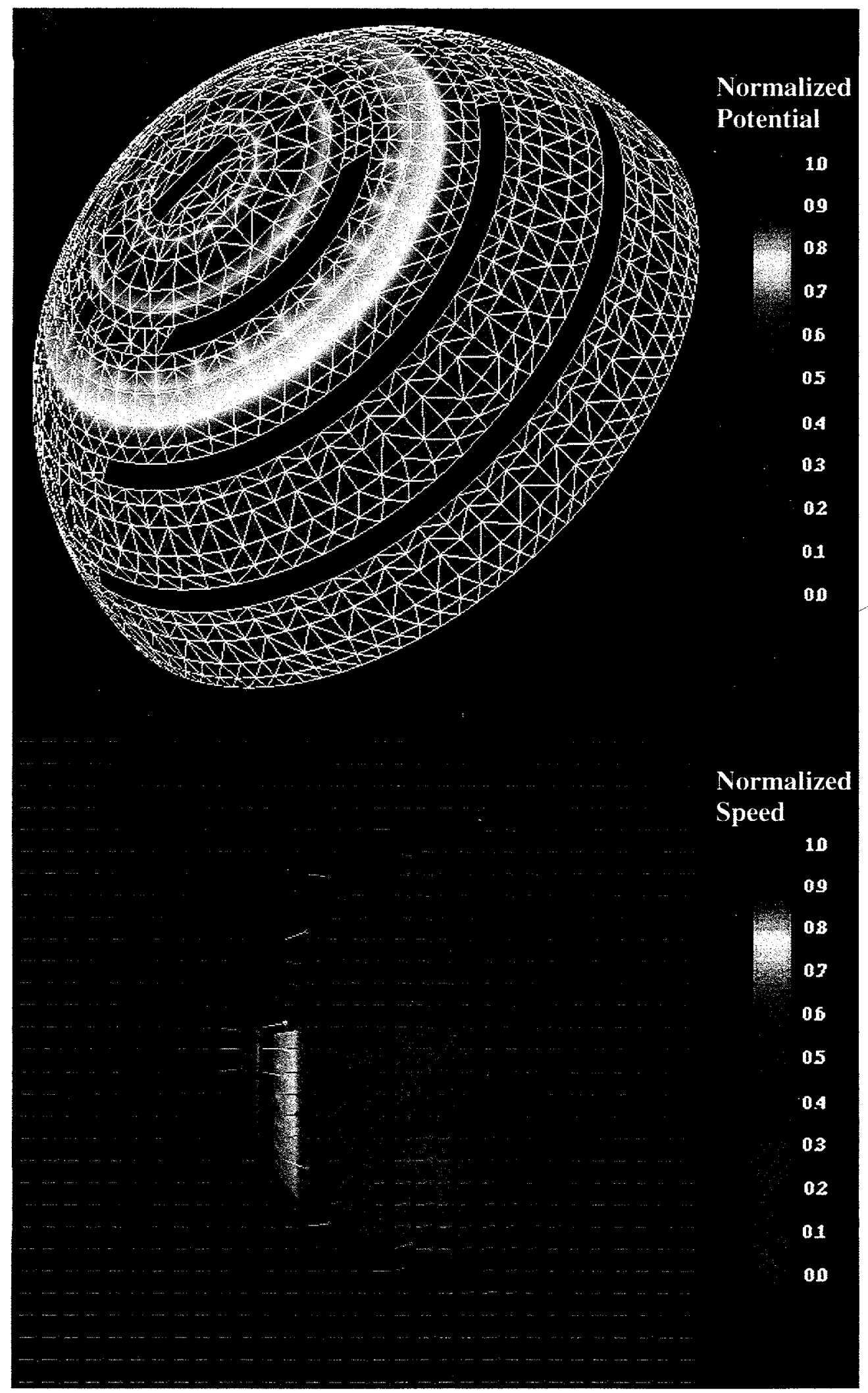

(a)

(b)

Figure 15 


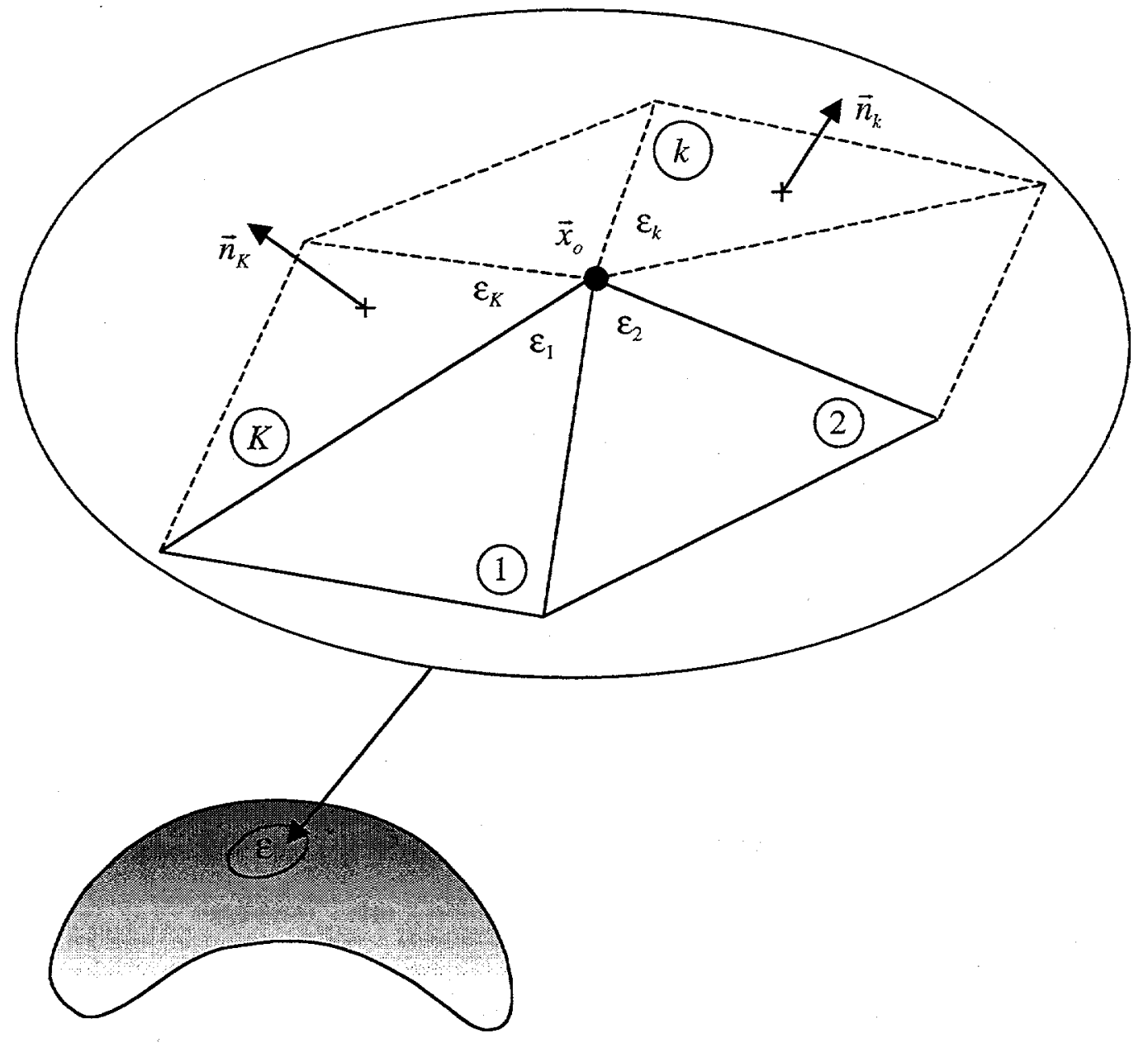

Figure B-1 


\section{APPENDIX A}

The regularized form of the potential velocity; i.e., Eq. (2.5), is derived in this section. We begin by the classical formulation, (2.4a), and concentrate on the integral quantity:

$$
\vec{\nabla}_{o} \int_{\partial \Omega}[\Phi] \vec{n} \cdot \vec{\nabla} G d S \equiv \int_{\partial \Omega}[\Phi] \vec{n} \cdot \vec{\nabla} \vec{g}_{o} d S
$$

where $\vec{g}_{o} \equiv \vec{\nabla}_{o} G$.

We apply the vector identity $(\vec{n} \times \vec{\nabla}) \times \vec{g}_{o}-\vec{n} \times\left(\vec{\nabla} \times \vec{g}_{o}\right) \equiv(\vec{n} \cdot \vec{\nabla}) \vec{g}_{o}-\vec{n}\left(\vec{\nabla} \cdot \vec{g}_{o}\right)[15]$ to the above, and note that the second terms on the two sides of the identity vanish:

$$
\begin{aligned}
\int_{\partial \Omega}[\Phi] \vec{n} \cdot \vec{\nabla} \vec{g}_{o} d S & \equiv \int_{\partial \Omega}[\Phi](\vec{n} \times \vec{\nabla}) \times \vec{g}_{o} d S \\
& \equiv \int_{\partial \Omega}(\vec{n} \times \vec{\nabla}) \times\left([\Phi] \vec{g}_{o}\right) d S-\int_{\partial \Omega}(\vec{n} \times \vec{\nabla}[\Phi]) \times \vec{g}_{o} d S
\end{aligned}
$$

Applying Stokes Theorem to the first integral on the right hand side, we get

$$
\int_{\partial \Omega}[\Phi] \vec{n} \cdot \vec{\nabla} \vec{g}_{o} d S \equiv-\oint_{\partial \Omega}[\Phi] \vec{g}_{o} \times d \vec{s}-\int_{\partial \Omega}(\vec{n} \times \vec{\nabla}[\Phi]) \times \vec{g}_{o} d S
$$

Substituting $\vec{g}_{o} \equiv \vec{\nabla}_{o} G$ in the above we get the desired regularized integral equation as follows:

$$
\vec{\nabla}_{o} \int_{\partial \Omega}[\Phi] \vec{n} \cdot \vec{\nabla} G d S \equiv \vec{\nabla}_{o} \times\left\{\int_{\partial \Omega}(\vec{n} \times \vec{\nabla}[\Phi]) G d S-\oint_{\partial \Omega}[\Phi] G d s\right\}
$$




\section{APPENDIX B}

We show here that the left hand side of Eq. (2.11) is singular unless $[\Phi]$ is $C^{1}$ continuous at the collocation points:

$$
\vec{n}_{o} \cdot\left[\vec{\nabla}_{o} \times \int_{\partial \Omega}(\vec{n} \times \vec{\nabla}[\Phi]) G d S\right] \equiv \vec{n}_{o} \cdot \int_{\partial \Omega}(\vec{n} \times \vec{\nabla}[\Phi]) \times \vec{\nabla} G d S
$$

where $\vec{\nabla} G \equiv-\vec{\nabla}_{o} G$ was used. The right hand side of (B.1) may be reformulated as

$$
\begin{aligned}
\vec{n}_{o} \cdot \int_{\partial \Omega}(\vec{n} \times \vec{\nabla}[\Phi]) \times \vec{\nabla} G d S & \equiv \int_{\partial \Omega} \vec{n}_{o} \cdot \vec{\nabla} \times(\vec{n} \times \vec{\nabla}[\Phi]) G d S \\
& -\int_{\partial \Omega} \vec{n}_{o} \cdot \vec{\nabla} \times[(\vec{n} \times \vec{\nabla}[\Phi]) G] d S
\end{aligned}
$$

The first integral on the right hand side of (B.2) is regular and presents no difficulties. To analyze the behavior of the second integral at the collocation point $\vec{x}_{o}$, we assume - without loss of generality - that the surface of an arbitrarily small area $\varepsilon$ around the collocation point is discretized using $K$ plane triangular elements such that $\varepsilon=\sum_{k=1}^{K} \varepsilon_{k}$ and that the elements share $\vec{x}_{o}$ (see Fig. B.1). As a result, since the unit normal is constant for planar elements, we conclude that $\vec{n}_{o, k}=\vec{n}_{k}$ for each triangle. Therefore, we can rewrite the second integral in Eq. (B.2) as

$$
\begin{aligned}
\int_{\partial \Omega} \vec{n}_{o} \cdot \vec{\nabla} \times[(\vec{n} \times \vec{\nabla}[\Phi]) G] d S & =\int_{\partial \Omega \backslash \varepsilon} \vec{n}_{o} \cdot \vec{\nabla} \times[(\vec{n} \times \vec{\nabla}[\Phi]) G] d S \\
& +\sum_{k=1}^{K} \int_{\varepsilon_{k}} \vec{n}_{k} \cdot \vec{\nabla} \times\left[(\vec{n} \times \vec{\nabla}[\Phi])_{k} G\right] d S_{k}
\end{aligned}
$$

and upon the application of Stokes Theorem to the second integral above:

$$
\begin{aligned}
\int_{\partial \Omega} \vec{n}_{o} \cdot \vec{\nabla} \times[(\vec{n} \times \vec{\nabla}[\Phi]) G] d S= & \int_{\partial \Omega \backslash \varepsilon} \vec{n}_{o} \cdot \vec{\nabla} \times[(\vec{n} \times \vec{\nabla}[\Phi]) G] d S \\
& +\sum_{k=1}^{K} \oint_{\varepsilon_{k}} G(\vec{n} \times \vec{\nabla}[\Phi])_{k} \cdot d \vec{s}_{k}
\end{aligned}
$$

The first integral on the right hand side above is regular, since it excludes the collocation point $\vec{x}_{o}$. However, the line integral is singular at $\vec{x}_{o}$ unless $\vec{n} \times \vec{\nabla}[\Phi]$ is at least $C^{0}$ continuous there, in which case the contributions to the line integrals by the neighboring elements will lead to the exact cancellation of the singularity. Therefore, for collocation based computations, Eq. (2.11) is useful only if $[\Phi]$ is $C^{1}$ continuous at the collocation nodes. Furthermore, under such a condition the second integral on the right hand side of (B.2) vanishes at the collocation points and (2.11) reduces to the following regularized integral equation:

$$
\int_{\partial \Omega} \vec{n}_{o} \cdot \vec{\nabla} \times(\vec{n} \times \vec{\nabla}[\Phi]) G d S=\vec{n}_{o} \cdot \vec{V}_{\infty}
$$




\section{APPENDIX C}

In this section we present the 2-D RGBEM formulation as a special case of Eq. (2.15). For this purpose, we use the same local orthonormal coordinate system as in the 3-D case, with unit basis vector $(\vec{\tau}, \vec{\rho}, \vec{n})$. For the 2-D case, however, $\vec{\tau}=\left(\tau_{x}, \tau_{y}\right)$ is parallel to the body contour and $\vec{n}=\left(n_{x}, n_{y}\right)$ is normal to it. $\vec{\rho}$ is normal to the computational domain. To maintain the same nomenclature as before, we specify the independent variables in the local coordinate system by $(\xi, \eta)$. The tangential gradient of a dummy variable $f$ representing $[\Phi]$ or $\psi$ is thus given by:

$$
\vec{n} \times \vec{\nabla} f=f_{, \xi} \vec{\rho}
$$

Using Eq. (C.1) and recognizing that the integrals in Eq. (2.15) are line (and not surface) integrals, we are in the position to present the final form of the 2-D RGBEM formulation:

$$
\int_{\partial \Omega_{o}} \int_{\partial \Omega} \frac{\partial \psi_{o}}{\partial \xi_{o}} \frac{\partial[\Phi]}{\partial \xi} G d \xi d \xi_{o}=-\int_{\partial \Omega_{o}} \vec{n}_{o} \cdot \vec{V}_{\infty} \psi_{o} d \xi_{o}
$$

where $G\left(\vec{x}, \vec{x}_{o}\right)=-1 / 2 \pi \ln \left|\vec{x}-\vec{x}_{o}\right|=-1 / 2 \pi \ln r$ is the 2-D Green function. Note that, in (C.2), we have assumed $\vec{\rho} \cdot \vec{\rho}_{o}=1$, which is valid so long as $(\vec{\tau}, \vec{n})$ are assigned consistently around the body contour.

As in the 3-D case, Eq. (C.2) simplifies substantially if the body contour is discretized using $M$ linear segments, and if $[\Phi]^{m}$ and $\psi^{m}$ on element $m$ are assumed to vary linearly in the form $f\left(\xi^{m}\right)=a_{f}^{m}+b_{f}^{m} \xi^{m}$, where

$$
\begin{aligned}
& a_{f}^{m}=\frac{\xi_{2}^{m} f_{1}^{m}-\xi_{1}^{m} f_{2}^{m}}{L_{m}} \\
& b_{f}^{m}=\frac{f_{2}^{m}-f_{1}^{m}}{L_{m}}
\end{aligned}
$$

$L_{m}=\xi_{2}^{m}-\xi_{1}^{m}$ is the length of segment $m$, and indices 1 and 2 point to the beginning and the end of the segment, respectively. Note that $\left(f_{, \xi}\right)_{m}=b_{f}^{m}$. Therefore, substituting Eq. (C.3b) in the discretized form of Eq. (C.2) yields the linear 2D RGBEM formulation:

$$
\sum_{k=1}^{M} \sum_{m=k}^{M}\left(\psi_{2}^{k}-\psi_{1}^{k}\right)\left([\Phi]_{2}^{m}-[\Phi]_{1}^{m}\right) I_{m k}=-\sum_{k=1}^{M} \int_{\xi_{1}^{k}}^{\xi_{k}^{k}} \vec{n}_{k} \cdot \vec{V}_{\infty}^{k}\left(a_{\psi}^{k}+b_{\psi}^{k} \xi^{k}\right) d \xi^{k}
$$

where $I_{m k}=\int_{\xi_{1}^{k}}^{\xi_{2}^{k}} \int_{\xi_{1}^{m}}^{\xi_{2}^{m}} G \frac{d \xi^{m}}{L_{m}} \frac{d \xi^{k}}{L_{k}}$. 
For piecewise-uniform free stream velocity $\vec{V}_{\infty}^{k}$ across each element $k$, the linear system of $N$ equations for the unknown [ $\Phi]$ at the $N$ collocation points is given as follows:

$$
\begin{aligned}
& \sum_{k=1}^{M} \sum_{m=k}^{M} \sum_{i=1}^{2} \sum_{j=1}^{2} A_{i j}^{m k}[\Phi]_{i}^{m} \psi_{j}^{k}=\sum_{k=1}^{M} \sum_{j=1}^{2} B_{k} \psi_{j}^{k} \\
& A_{i j}^{m k}=P_{i} P_{j} \int_{\xi_{1}^{k}}^{\xi_{2}^{k}} \int_{\xi_{1}^{m}}^{\xi_{2}^{m}} G \frac{d \xi^{m}}{L_{m}} \frac{d \xi^{k}}{L_{k}} \\
& B_{k}=-\frac{1}{2} \vec{n}_{k} \cdot \vec{V}_{\infty}^{k} L_{k} \\
& P_{1}=1 \quad, \quad P_{2}=-1
\end{aligned}
$$

where the process of matrix assembly follows the discussion in Section 2.3.

For coincident elements where $m=k$, the double line integral is easily evaluated as

$$
I_{k k}=-\frac{1}{4 \pi}\left(2 \log L_{k}-3\right)
$$

For adjacent elements the integrals are a bit more complicated:

$$
\begin{aligned}
& I_{m k}=-\frac{1}{4 \pi}\left\{\begin{array}{l}
S_{m k} *\left[\frac{L_{m}}{L_{k}} \tan ^{-1}\left(\frac{S_{m k}}{L_{m} / L_{k}+C_{m k}}\right)+\frac{L_{k}}{L_{m}} \tan ^{-1}\left(\frac{S_{m k}}{L_{k} / L_{m}+C_{m k}}\right)\right]+ \\
C_{m k} *\left[\frac{L_{m}}{L_{k}} \log \left(\frac{L_{m k}}{L_{m}}\right)+\frac{L_{k}}{L_{m}} \log \left(\frac{L_{m k}}{L_{k}}\right)\right]+2 \log L_{m k}-3
\end{array}\right\} \\
& C_{m k}=\bar{\tau}_{m} \cdot \bar{\tau}_{k} \quad, \quad S_{m k}=\sqrt{1-\left(C_{m k}\right)^{2}} \\
& L_{m k}^{2}=L_{m}^{2}+L_{k}^{2}+2 C_{m k} L_{m} L_{k}
\end{aligned}
$$

The remaining integrals may be evaluated analytically for the inner integral and numerically for the outer one. 


\section{DISTRIBUTION}

2 Analytical Methods, Inc.

$2133152^{\text {nd }}$ Ave.

Redmond, WA 98052

Attn: F. Dvorak

B. Maskew

1 Prof. A. J. Chorin

Department of Mathematics

University of California

Berkeley, CA 94720

1 L. Cortelezzi

UCLA

Dept. of Mathematics

Los Angeles, CA 90095-1555

1 Dr. R. Cortez

Dept. of Mathematics

Tulane University

6823 St. Charles Ave, \#424

New Orleans, LA 70118

25 Dr. Adrin Gharakhani Applied Scientific Research 1800 E. Garry Ave., Suite 214

Santa Ana, CA 92705

1 Prof. A. F. Ghoniem

Massachusetts Institute of Technology

Mechanical Engineering Dept.

Room 3-342

Cambridge, MA 02139-4307

1 Dr. John R. Grant

Naval Undersea Center

1176 Howell Street

Building 108, Code 8233

Newport, RI 02841-1708

1 Dr. Leonard J. Gray

Oak Ridge National Laboratory

P.O. Box 2008, Bldg. 6012

Oak Ridge, TN 37831-6367
1 Prof. C. E. Hailey

Mech. \& Aero. Eng

Utah State University

UMC 4130

Logan, UT 84322-4130

1 Prof. H. Higuchi

Dept. of Mech. \& Aero. Eng.

Syracuse University

Syracuse, NY 13244

1 Dr. Stephen Huyer

Naval Undersea Weapon Center

Hydrodynamics Branch, Code 8233

Building 1302/1

Newport, RI 02841

1 Dr. Vinay Kalro

Fluent, Inc.

1508 Oak Ave., \#3N

Evanston, IL 60201

1 Prof. A. R. Karagozian

UCLA

46-147D Engr. IV

Mail Code 951597

Los Angeles, CA 90095-1597

1 Prof. Joseph Katz

Dept. Aerospace Eng. and Eng. Mech.

San Diego State University

San Diego, CA 92182-0183

1 Prof. Omar M. Knio

Dept. of Mech. Eng.

Johns Hopkins University

Baltimore, MD 21218-2686

1 Prof. Anthony Leonard

Graduate Aeronautics Lab.

California Institute of Technology

Pasadena, CA 91125 
1 Prof. John Leonard Dept. of Civil \& Env. Eng. University of Connecticut Storrs, CT 06269-2037

1 Dr. William W. Liou Dept. of Mech. and Aero. Engineering Western Michigan University Kalamazoo, MI 49008

1 Prof. Mark D. Maughmer Department of Aerospace Engineering The Pennsylvania State University University Park, PA 16802

1 Prof. Jeffery S. Marshall Iowa Institute of Hydraulic Research University of Iowa 300 S. Riverside Drive Iowa City, IA 52242-1585

1 Dr. Eckart Meiburg Dept. of Aerospace Eng. University of Southern California 854 W. 36th Place Los Angeles, CA 90089-1191

1 Prof. D. I. Meiron

Dept. of Applied Mathematics California Institute of Technology Pasadena, CA 91125

1 Dr. M. Nitsche Dept. of Math. \& Stat. University of New Mexico Albuquerque, NM 87131

1 Prof. P. G. Saffman Dept. of Applied Mathematics California Institute of Technology Pasadena, CA 91125
1 Prof. William Saric

Dept of Mech. and Aerospace Eng. Arizona State University

PO Box 876106

Tempe, AZ 85287-6106

1 Prof. T. Sarpkaya

Dept. Mech. Eng.

Code 69-SL

Naval Postgraduate Academy

Monterey, CA 93943

1 Prof. Michael S. Selig

Dept. of Aero. and Astro. Engineering

306 Talbot Lab

Urbana, IL 61801

1 Prof. J. A. Sethian

Dept. of Mathematics

University of California

Berkeley, CA 94720

1 Prof. Marios C. Soteriou

Dept. of Mech. Eng.

University of Connecticut

191 Auditorium Rd., U-139

Storrs, CT 06269-3139

1 Prof. J. A. Strain

Dept. of Mathematics

University of California

Berkeley, CA 94720

1 Dr. S. Subramaniam

Massachusetts Institute of Technology

Mechanical Engineering Dept.

Room 3-339

Cambridge, MA 02139

1 Prof. G. Trygvasson

Dept. Mech. Eng. and App. Mech.

University of Michigan

Ann Arbor, MI 48109-2125 
2 University of New Mexico

Dept. of Mech. Eng.

Albuquerque, NM 87131

Attn: M. S. Ingber

C. R. Truman

1 Dr. James Uhlman

Code 804, Bldg. 108/2

Naval Undersea Warfare Center

Newport, RI 02841-5047

2 U.S. Army Soldier \& Biological Chemical Command

Soldier Systems Center Natick

Kansas Street

Natick, MA 01760-5017

ATTN: SSCNC-UTS (Richard Benney)

SSCNC-UTS (Keith Stein)

1 Prof. C. W. Van Atta

Dept. of App. Mech. and Eng. Sci.

Mail Code 0411

University of California

La Jolla, CA 92093-0411

1 Prof. L. Van Dommelen

Dept. of Mech. Eng.

Florida State University

PO Box 2175

Tallahassee, FL 32316-2175

3 Washington State University

Dept. Mech. \& Matl's Eng.

Pullman, WA 99164-2920

Attn: Jacob Chung Clayton Crowe

Tim Troutt

1 Prof. R. E. Wilson

Dept. Mech. Eng.

Oregon State University

Corvallis, OR 97331
1 Prof. Norman J. Zabusky

Dept. of Mech. and Aerospace Eng.

Rutgers University

PO Box 909

Piscataway, NJ 08855-0909

1 Dr. David Zielke

Naval Research Lab, 6-4-40

4555 Overlook Avenue, SW

Washington, DC 20375

1 Dr. P. W. Bearman

Dept. of Aeronautics

Imperial College

London, SW7 2BY

ENGLAND

1 Prof. Ion Paraschiviou

Dept. of Mech. Eng.

Ecole Polytecnique

CP 6079

Succursale A

Montreal H3C 3A7, CANADA

1 Dr. David Sharpe

Department of Aeronautical Eng.

Queen Mary College

Mile End Road

London, E1 4NS

ENGLAND

1 Prof. G. S. Wincklemans

Center for Sys. Eng. \& App. Mech.

Unité TERM, place du Levant 2 , Université Catholique de Louvain Louvain-la-Neuve 1348

BELGIUM 
0708 D. E. Berg, 6214

0708 H. M. Dodd, 6214

0825 F. G. Blottner, 9115

0825 V. L. Bahr, 9115

0825 W. H. Rutledge, 9115

0826 W. L. Hermina, 9113

0827 R. Griffith, 9117

0828 J. A. Fernandez, 9102

0828 K. Radloff, 9102

0828 R. K. Thomas, 9102

0828 R. S. Baty, 9133

0828 T. C. Bickel, 9101

0828 W. L. Oberkampf, 9133

0834 A. C. Ratzel, 9112

0835 A. A. Gossler, 9111

0835 G. F. Homicz, 9111

0835 J. H. Strickland, 9111

0835 J. S. Peery, 9121

0835 M. W. Glass, 9111

0835 R. J. Cochran, 9111

0835 S. Burns, 9111

0835 S. N. Kempka, 9111

0835 S. R. Subia, 9111

0835 V. L. Porter, 9121

0835 W. P. Wolfe, 9111

0836 C. E. Hickox, 9116

0836 C. W. Peterson, 9100

0836 L. A. Gritzo, 9116

0836 S. R. Tieszen, 9116

0841 P. J. Hommert, 9100

9051 H. N. Najm, 8351

9051 L. A. Rahn, 8351

9051 W. T. Ashurst, 8351

9018 Central Technical Files, 8940-2

20899 Technical Library, 4916

10612 Review \& Approval Desk, 4912, For DOE/OSTI 